\title{
Geometry of submanifolds with respect to ambient vector fields
}

\author{
Fernando Manfio, Ruy Tojeiro and Joeri Van der Veken *
}

2010 Mathematics Subject Classification: 53 B25.

Key words and phrases: Constant ratio property, Principal direction property, Radial vector field, Killing vector field, conformal Killing vector field, loxodromic isometric immersion.

\begin{abstract}
Given a Riemannian manifold $N^{n}$ and $\mathcal{Z} \in \mathfrak{X}(N)$, an isometric immersion $f: M^{m} \rightarrow N^{n}$ is said to have the constant ratio property with respect to $\mathcal{Z}$ either if the tangent component $\mathcal{Z}_{f}^{T}$ of $\mathcal{Z}$ vanishes identically or if $\mathcal{Z}_{f}^{T}$ vanishes nowhere and the ratio $\left\|\mathcal{Z}_{f}^{\perp}\right\| /\left\|\mathcal{Z}_{f}^{T}\right\|$ between the lengths of the normal and tangent components of $\mathcal{Z}$ is constant along $M^{m}$. It has the principal direction property with respect to $\mathcal{Z}$ if $\mathcal{Z}_{f}^{T}$ is an eigenvector of all shape operators of $f$ at all points of $M^{m}$. In this article we study isometric immersions $f: M^{m} \rightarrow N^{n}$ of arbitrary codimension that have either the constant ratio or the principal direction property with respect to distinguished vector fields $\mathcal{Z}$ on space forms, product spaces $\mathbb{S}^{n} \times \mathbb{R}$ and $\mathbb{H}^{n} \times \mathbb{R}$, where $\mathbb{S}^{n}$ and $\mathbb{H}^{n}$ are the $n$-dimensional sphere and hyperbolic space, respectively, and, more generally, on warped products $I \times{ }_{\rho} \mathbb{Q}_{\epsilon}^{n}$ of an open interval $I \subset \mathbb{R}$ and a space form $\mathbb{Q}_{\epsilon}^{n}$. Starting from the observation that these properties are invariant under conformal changes of the ambient metric, we provide new characterizations and classification results of isometric immersions that satisfy either of those properties, or both of them simultaneously, for several relevant instances of $\mathcal{Z}$ as well as simpler descriptions and proofs of some known ones for particular cases of $\mathcal{Z}$ previously considered by many authors. Our methods also allow us to classify Euclidean submanifolds with the property that the normal components of their position vector fields are parallel with respect to the normal connection, and to give alternative descriptions to those in [3] of Euclidean submanifolds whose tangent or normal components of their position vector fields have constant length.
\end{abstract}

${ }^{*}$ Parts of this work were carried out while J. Van der Veken visited Universidade de São Paulo in the framework of the Young Researchers Summer Program 2018, and while Ruy Tojeiro visited the KULeuven partially supported by Aucani (USP) Public Notice 967/2018. J. Van der Veken is supported by project 3E160361 of the KU Leuven Research Fund and by EOS project G0H4518N of the Belgian government. Ruy Tojeiro is supported by Fapesp grant 2016/23746-6 and CNPq grant 303002/2017-4. 


\section{Introduction}

Let $M^{m}$ and $N^{n}$ be Riemannian manifolds of dimensions $m$ and $n$, respectively. Given a vector field $\mathcal{Z}$ on $N^{n}$, it is a natural problem to investigate the isometric immersions $f: M^{m} \rightarrow N^{n}$ that have relevant geometric properties with respect to $\mathcal{Z}$.

For example, for which oriented hypersurfaces $f: M^{m} \rightarrow N^{m+1}$ does a unit normal vector field along $f$ make a constant angle with $\mathcal{Z}$ ? A related problem is to look for the hypersurfaces for which the tangent component of $\mathcal{Z}$ is a principal direction of $f$ at any point of $M^{m}$.

Equivalent versions of these problems can also be posed for isometric immersions $f: M^{m} \rightarrow N^{n}$ of arbitrary codimension $n-m$. Namely, the first one is equivalent to looking for the hypersurfaces for which either the tangent component of $\mathcal{Z}$ vanishes everywhere or it is nowhere vanishing and the ratio between the lengths of the normal and tangent components of $\mathcal{Z}$ is constant, and in this form the problem makes sense for submanifolds of arbitrary codimension. One may also consider the second of the preceding problems for submanifolds of any codimension by requiring the tangent component of $\mathcal{Z}$ to be a principal direction of all shape operators of $f$. It will be convenient to refer to isometric immersions satisfying those conditions as isometric immersions having the constant ratio property or the principal direction property with respect to $\mathcal{Z}$, respectively.

These problems are particularly interesting when $N^{n}$ is endowed with distinguished vector fields $\mathcal{Z}$, for instance if $N^{n}$ is a space form or a Riemannian product $\mathbb{Q}_{\epsilon}^{n} \times \mathbb{R}$, where $\mathbb{Q}_{\epsilon}^{n}$ denotes either the sphere $\mathbb{S}^{n}$ or the hyperbolic space $\mathbb{H}^{n}$ of dimension $n$, depending on whether $\epsilon=1$ or $\epsilon=-1$, respectively. More generally, if $N^{n}$ is a warped product $I \times{ }_{\rho} P$ of an open interval $I \subset \mathbb{R}$ with any Riemannian manifold $P$. In particular, in Euclidean space one may consider the above problems when $\mathcal{Z}$ is either a constant or the radial vector field, or when it is a Killing or a conformal Killing vector field. In a product space $\mathbb{Q}_{\epsilon}^{n} \times \mathbb{R}$ or, more generally, in any warped product $I \times_{\rho} P$, a natural vector field $\mathcal{Z}$ to consider is a unit vector field $\frac{\partial}{\partial t}$ tangent to either the factor $\mathbb{R}$ or the factor $I$, respectively. One may also consider those problems for the "radial" vector fields in $\mathbb{Q}_{\epsilon}^{n}$, that is, the closed and conformal vector fields in those spaces.

Several authors have addressed particular instances of the above problems for special cases of the ambient space $N^{n}$ and of the vector field $\mathcal{Z}$. In particular, hypersurfaces with the constant ratio property with respect to the unit vector field $\mathcal{Z}=\frac{\partial}{\partial t}$ tangent to the factor $\mathbb{R}$ of a product space $\mathbb{S}^{n-1} \times \mathbb{R}$ and $\mathbb{H}^{n-1} \times \mathbb{R}$, or with respect to a constant vector field in Euclidean space, were described in [5], [7] and [19], and submanifolds with the principal direction property with respect to those vector fields in [6], [8], [15] and [19]. The first of these problems was studied in [12] for surfaces in a warped product $I \times_{\rho} \mathbb{R}^{2}$ and for hypersurfaces of arbitrary dimension of any warped product $I \times_{\rho} P$ in [11]. The constant ratio property with respect to the radial vector field in Euclidean space was investigated in [16] for surfaces in $\mathbb{R}^{3}$, for Euclidean hypersurfaces 
of any dimension in [1] and [21], and for submanifolds of arbitrary codimension in [2]. Surfaces in $\mathbb{R}^{3}$ with the constant ratio property with respect to a Killing vector field were studied in [18]. The principal direction property with respect to the radial vector field was investigated in [17] for surfaces in $\mathbb{R}^{3}$ and with respect to the vector field $\mathcal{Z}=\frac{\partial}{\partial t}$ tangent to the factor $\mathbb{R}$ for hypersurfaces of a warped product $\mathbb{R} \times_{\rho} P$ in [10]. Constant mean curvature hypersurfaces of $\mathbb{R} \times{ }_{\rho} P$ with this property were characterized in [9], yielding a description of constant mean curvature hypersurfaces with the principal direction property with respect to closed and conformal vector fields in $\mathbb{Q}_{\epsilon}^{n}$.

Our first main result is a description of the isometric immersions $f: M^{m} \rightarrow \mathbb{R}^{n}$, for arbitrary values of the dimension $m$ and the codimension $n-m$, that have the constant ratio property with respect to a constant vector field, as well as of the isometric immersions $f: M^{m} \rightarrow \mathbb{Q}_{\epsilon}^{n} \times \mathbb{R}$ that have the constant ratio property with respect to the unit vector field $\mathcal{Z}=\frac{\partial}{\partial t}$ tangent to the factor $\mathbb{R}$.

Several of the remaining results of this article rely on the elementary but useful observation that, for an isometric immersion $f: M^{m} \rightarrow N^{n}$, both the principal direction and constant ratio properties with respect to a vector field $\mathcal{Z}$ on $N^{n}$ are invariant under conformal changes of the metric of the ambient space. Equivalently, if $\Psi: N^{n} \rightarrow \hat{N}^{n}$ is a (local) conformal diffeomorphism, then any of these properties holds for an isometric immersion $f: M^{m} \rightarrow N^{n}$ with respect to $\mathcal{Z} \in \mathfrak{X}(N)$ if and only if it holds for $\hat{f}=\Psi \circ f$ with respect to $\hat{\mathcal{Z}}=\Psi_{*} \mathcal{Z} \in \mathfrak{X}(\hat{N})$.

By applying the preceding observation, we first extend the description of isometric immersions $f: M^{m} \rightarrow \mathbb{Q}_{\epsilon}^{n} \times \mathbb{R}$ with the constant ratio property with respect to the unit vector field $\mathcal{Z}=\frac{\partial}{\partial t}$ tangent to the factor $\mathbb{R}$ to the case in which the ambient space is any warped product $I \times{ }_{\rho} \mathbb{Q}_{\epsilon}^{n}$, with $I \subset \mathbb{R}$ an open interval, making use of the fact that any warped product metric on $I \times \mathbb{Q}_{\epsilon}^{n}$ is conformal to the standard Riemannian product metric. We then use the well known representations of (open dense subsets of) $\mathbb{Q}_{\epsilon}^{n+1}$ as such a warped product to describe all "loxodromic" isometric immersions into a space form $\mathbb{Q}_{\epsilon}^{n+1}$, that is, isometric immersions into $\mathbb{Q}_{\epsilon}^{n+1}$ that have the constant ratio property with respect to a "radial" vector field in $\mathbb{Q}_{\epsilon}^{n+1}$.

Then we apply the same basic observation to the well-known conformal diffeomorphism of $\mathbb{S}^{n-1} \times \mathbb{R}$ onto $\mathbb{R}^{n} \backslash\{0\}$, and give a similar description of all isometric immersions $f: M^{m} \rightarrow \mathbb{R}^{n} \backslash\{0\}$ that have the constant ratio property with respect to the radial vector field, making the classification in [2] more explicit. In the hypersurface case we recover the main result of [21] (see also [1] and [16]).

We use the same idea to classify isometric immersions $f: M^{m} \rightarrow \mathbb{R}^{n} \backslash\{0\}$ that have the principal direction property with respect to the radial vector field, extending for arbitrary values of $m$ and $n$ the main result of [17], where this problem was studied for surfaces in $\mathbb{R}^{3}$. We also determine the isometric immersions $f: M^{m} \rightarrow \mathbb{R}^{n} \backslash\{0\}$ that have both the constant ratio and principal direction properties with respect to the radial vector field.

Our methods also allow us to classify the related class of Euclidean submanifolds with 
the property that the normal components of their position vector fields are parallel with respect to the normal connection, and to give an alternative description of Euclidean submanifolds whose tangent or normal components of their position vector fields have constant length. The latter were first described in [3], where they were called $T$-constant and $N$-constant submanifolds.

Our description of isometric immersions $f: M^{m} \rightarrow \mathbb{R}^{n} \backslash\{0\}$ that have the constant ratio property with respect to the radial vector field is thus based on the observation that any such isometric immersion is the composition $f=\Psi \circ \hat{f}$ of an isometric immersion $\hat{f}: M^{m} \rightarrow \mathbb{S}^{n-1} \times \mathbb{R}$ that has the constant ratio property with respect to $\frac{\partial}{\partial t}$ with the conformal diffeomorphism $\Psi: \mathbb{S}^{n-1} \times \mathbb{R} \rightarrow \mathbb{R}^{n} \backslash\{0\}$. Notice that in the very particular case in which $m=1$ and $n=2$, that is, unit speed curves in $\mathbb{R}^{2}$, our approach reduces to the observation that the logarithmic spiral, the only unit speed plane curve that has the constant ratio property with respect to the radial vector field (with nonvanishing tangent and normal components of that vector field), is just the image under the conformal diffeomorphism of $\mathbb{S}^{1} \times \mathbb{R}$ onto $\mathbb{R}^{2} \backslash\{0\}$ of a circular helix in $\mathbb{S}^{1} \times \mathbb{R}$.

The corresponding conformal covering map of $\mathbb{H}^{n-1} \times \mathbb{R}$ onto $\mathbb{R}^{n} \backslash \mathbb{R}^{n-1}$ is then used to describe all isometric immersions $f: M^{m} \rightarrow \mathbb{R}^{n} \backslash\{0\}$ that have either the constant ratio or the principal direction property with respect to a Killing vector field $\mathcal{K}$ in $\mathbb{R}^{n}$ generating rotations around a subspace $\mathbb{R}^{n-1}$ of $\mathbb{R}^{n+1}$. The first of these problems was investigated in [18] for surfaces in $\mathbb{R}^{3}$. However, their classification misses a large class of examples (see Remark 24 below).

As one further application of our basic observation, we describe all isometric immersions into Euclidean space that have the constant ratio property or the principal direction property with respect to any of the vector fields that generate the Lie algebra of conformal Killing vector fields in $\mathbb{R}^{n}$.

\section{Some basic lemmas}

Given an isometric immersion $f: M^{m} \rightarrow N^{n}$ and $\mathcal{Z} \in \mathfrak{X}(N)$, we always denote by $\mathcal{Z}_{f}^{T} \in \mathfrak{X}(M)$ and $\mathcal{Z}_{f}^{\perp} \in \Gamma\left(N_{f} M\right)$ the tangent and normal vector fields, respectively, given by orthogonally decomposing $\mathcal{Z}$ into its tangent and normal components along $f$, i.e.,

$$
\mathcal{Z}(f(x))=f_{*}(x) \mathcal{Z}_{f}^{T}(x)+\mathcal{Z}_{f}^{\perp}(x) \text { for all } x \in M^{m} .
$$

Definition 1. Given a Riemannian manifold $N^{n}$ and $\mathcal{Z} \in \mathfrak{X}(N)$, we say that an isometric immersion $f: M^{m} \rightarrow N^{n}$ has

(i) the constant ratio property with respect to $\mathcal{Z}$ either if $\mathcal{Z}_{f}^{T}$ vanishes identically or if $\mathcal{Z}_{f}^{T}$ vanishes nowhere and $\left\|\mathcal{Z}_{f}^{\perp}\right\| /\left\|\mathcal{Z}_{f}^{T}\right\|$ is constant along $M^{m}$;

(ii) the principal direction property with respect to $\mathcal{Z}$ if $\mathcal{Z}_{f}^{T}$ is an eigenvector of all shape operators of $f$ at all points of $M^{m}$. 
Clearly, an isometric immersion $f: M^{m} \rightarrow N^{n}$ has either the constant ratio or the principal direction property with respect to $\mathcal{Z} \in \mathfrak{X}(N)$ if and only if it has the same property with respect to $\lambda \mathcal{Z}$ for some (and hence for any) $\lambda \in C^{\infty}(N)$ with no zeroes along $f(M)$.

In case $\mathcal{Z} \in \mathfrak{X}(N)$ is a parallel vector field in $N^{n}$, the next lemma gives equivalent conditions for an isometric immersion $f: M^{m} \rightarrow N^{n}$ to have the principal direction or the constant ratio property with respect to $\mathcal{Z}$, and in particular clarifies how these properties are related in this case. We denote by $\alpha_{f}$ the second fundamental form of $f$, by $A_{\xi}^{f}$ its shape operator with respect to $\xi \in \Gamma\left(N_{f} M\right)$, and by $\nabla^{\perp}$ its normal connection. Given a Riemannian manifold $M^{m}$ and a nowhere vanishing $X \in \mathfrak{X}(M)$, we denote by $\{X\}^{\perp}$ the distribution of codimension one in $M^{m}$ given by the orthogonal complements of $X(x)$ in each tangent space $T_{x} M, x \in M^{m}$.

Lemma 2. Let $N^{n}$ be a Riemannian manifold that admits a parallel vector field $\mathcal{Z}$ and let $f: M^{m} \rightarrow N^{n}$ be an isometric immersion. Then $f$ has the principal direction property with respect to $\mathcal{Z}$ if and only if $\mathcal{Z}_{f}^{T}$ is nowhere vanishing and $\mathcal{Z}_{f}^{\perp}$ is parallel in the normal connection along $\left\{\mathcal{Z}_{f}^{T}\right\}^{\perp}$. If $n=m+1$, this is equivalent to $\left\|\mathcal{Z}_{f}^{T}\right\|$ being a nonzero constant along $\left\{\mathcal{Z}_{f}^{T}\right\}^{\perp}$. Moreover, the following assertions are equivalent:

(i) $f$ has the constant ratio property with respect to $\mathcal{Z}$;

(ii) $\left\|\mathcal{Z}_{f}^{T}\right\|$ (or equivalently, $\left\|\mathcal{Z}_{f}^{\perp}\right\|$ ) is constant along $M^{n}$;

(iii) $A_{\mathcal{Z}_{f}}^{f} \mathcal{Z}_{f}^{T}=0$;

(iv) Either $\mathcal{Z}_{f}^{T}$ is everywhere vanishing or the integral curves of $\mathcal{Z}_{f}^{T}$ are geodesics.

In particular, if $n=m+1$ then the constant ratio property with respect to $\mathcal{Z}$ implies the principal direction property with respect to $\mathcal{Z}$, if the constant value of $\left\|\mathcal{Z}_{f}^{T}\right\|$ is nonzero.

Proof. Taking the tangent and normal components of $\tilde{\nabla}_{X} \mathcal{Z}=0$ for $X \in \mathfrak{X}(M)$, where $\tilde{\nabla}$ is the Levi-Civita connection of $N^{n}$, and using the Gauss and Weingarten equations of $f$, yield

$$
\nabla_{X} \mathcal{Z}_{f}^{T}=A_{\mathcal{Z}_{f}^{+}}^{f} X
$$

and

$$
\alpha_{f}\left(X, \mathcal{Z}_{f}^{T}\right)=-\nabla_{X}^{\perp} \mathcal{Z}_{f}^{\perp}
$$

where $\nabla$ is the Levi-Civita connection of $M^{m}$. The first assertion in the statement is an immediate consequence of (2), and the second one is clear, for $\mathcal{Z}$ has constant length. The latter observation also gives the equivalence between $(i)$ and (ii). By (2), conditions (ii) and (iii) are equivalent, and the latter holds if and only if $\nabla_{\mathcal{Z}_{f}^{T}} \mathcal{Z}_{f}^{T}=0$ by (1), which 
yields the equivalence between $(i i i)$ and $(i v)$. The last assertion is now an immediate consequence of either $(i i)$ or $(i i i)$.

The next lemma plays a key role in the sequel.

Lemma 3. Let $f: M^{m} \rightarrow N^{n}$ be an isometric immersion, let $\Psi: N^{n} \rightarrow \hat{N}^{n}$ be a local conformal diffeomorphism and set $\hat{f}=\Psi \circ f$. Let $\mathcal{Z}$ and $\hat{\mathcal{Z}}$ be vector fields on $N^{n}$ and $\hat{N}^{n}$ that are $\Psi$-related, that is, $\hat{\mathcal{Z}}(\Psi(y))=\Psi_{*}(y) \mathcal{Z}(y)$ for all $y \in N^{n}$. Then the following assertions hold:

(i) $\hat{\mathcal{Z}}_{\hat{f}}^{T}(x)=\mathcal{Z}_{f}^{T}(x)$ and $\hat{\mathcal{Z}}_{\hat{f}}^{\perp}(x)=\Psi_{*}(f(x)) \mathcal{Z}_{f}^{\perp}(x)$ for all $x \in M^{m}$;

(ii) if $\mathcal{Z}_{f}^{T}$ is nowhere vanishing, then the ratios $\left\|\mathcal{Z}_{f}^{\perp}\right\| /\left\|\mathcal{Z}_{f}^{T}\right\|$ and $\left\|\hat{\mathcal{Z}}_{\hat{f}}^{\perp}\right\| /\left\|\hat{\mathcal{Z}}_{\hat{f}}^{T}\right\|$ coincide;

(iii) $f$ has the constant ratio property with respect to $\mathcal{Z}$ if and only if $\hat{f}$ has the constant ratio property with respect to $\hat{\mathcal{Z}}$;

(iv) $f$ has the principal direction property with respect to $\mathcal{Z}$ if and only if $\hat{f}$ has the principal direction property with respect to $\hat{\mathcal{Z}}$.

Proof. The assertion in $i$ ) follows from

$$
\begin{aligned}
\hat{f}_{*}(x) \hat{\mathcal{Z}}_{\hat{f}}^{T}(x)+\hat{\mathcal{Z}}_{\hat{f}}^{\perp}(x) & =\hat{\mathcal{Z}}(\hat{f}(x)) \\
& =\Psi_{*}(f(x)) \mathcal{Z}(f(x)) \\
& =\Psi_{*}(f(x)) f_{*}(x) \mathcal{Z}_{f}^{T}(x)+\Psi_{*}(f(x)) \mathcal{Z}_{f}^{\perp}(x) \\
& =(\Psi \circ f)_{*}(x) \mathcal{Z}_{f}^{T}(x)+\Psi_{*}(f(x)) \mathcal{Z}_{f}^{\perp}(x) \\
& =\hat{f}_{*}(x) \mathcal{Z}_{f}^{T}(x)+\Psi_{*}(f(x)) \mathcal{Z}_{f}^{\perp}(x) .
\end{aligned}
$$

Now, if $\varphi \in C^{\infty}(N)$ is the conformal factor of $\Psi$, that is,

$$
\left\langle\Psi_{*} X, \Psi_{*} Y\right\rangle=\varphi^{2}(y)\langle X, Y\rangle
$$

for all $y \in N^{n}$ and $X, Y \in T_{y} N$, then

$$
\begin{aligned}
\left\langle\hat{\mathcal{Z}}_{\hat{f}}^{T}(x), \hat{\mathcal{Z}}_{\hat{f}}^{T}(x)\right\rangle_{\hat{f}} & =\left\langle\hat{f}_{*}(x) \hat{\mathcal{Z}}_{\hat{f}}^{T}(x), \hat{f}_{*}(x) \hat{\mathcal{Z}}_{\hat{f}}^{T}(x)\right\rangle \\
& =\left\langle\Psi_{*}(f(x)) f_{*}(x) \mathcal{Z}_{f}^{T}(x), \Psi_{*}(f(x)) f_{*}(x) \mathcal{Z}_{f}^{T}(x)\right\rangle \\
& =\left(\varphi(f(x))^{2}\left\langle f_{*}(x) \mathcal{Z}_{f}^{T}(x), f_{*}(x) \mathcal{Z}_{f}^{T}(x)\right\rangle\right. \\
& =\left(\varphi(f(x))^{2}\left\langle\mathcal{Z}_{f}^{T}(x), \mathcal{Z}_{f}^{T}(x)\right\rangle_{f}\right.
\end{aligned}
$$

and

$$
\begin{aligned}
\left\langle\hat{\mathcal{Z}}_{\hat{f}}^{\perp}(x), \hat{\mathcal{Z}}_{\hat{f}}^{\perp}(x)\right\rangle & =\left\langle\Psi_{*}(f(x)) \mathcal{Z}_{f}^{\perp}(x), \Psi_{*}(f(x)) \mathcal{Z}_{f}^{\perp}(x)\right\rangle \\
& =\left(\varphi(f(x))^{2}\left\langle\mathcal{Z}_{f}^{\perp}(x), \mathcal{Z}_{f}^{\perp}(x)\right\rangle\right.
\end{aligned}
$$


for all $x \in M^{m}$, where $\langle,\rangle_{f}$ and $\langle,\rangle_{\hat{f}}$ denote the metrics induced by $f$ and $\hat{f}$, respectively. The assertion in (ii) is an immediate consequence of the preceding relations, and $(i i i)$ follows by combining $(i)$ and $(i i)$. Finally, $(i v)$ follows from the relation

$$
\alpha_{\hat{f}}(X, Y)=\Psi_{*} \alpha_{f}(X, Y)-\frac{1}{\varphi}\langle X, Y\rangle \Psi_{*}(\operatorname{grad} \varphi)^{\perp}
$$

between the second fundamental forms $\alpha_{f}, \alpha_{\hat{f}}$ of $f$ and $\hat{f}$, respectively.

As a consequence of Lemmas 2 and 3, for a hypersurface $f: M^{m} \rightarrow N^{m+1}$ the constant ratio property with respect to $\mathcal{Z} \in \mathfrak{X}(N)$ implies the principal direction property with respect to $\mathcal{Z}$ (if $\mathcal{Z}_{f}^{T}$ is nowhere vanishing) also when $\mathcal{Z}$ is $\Psi$-related to a parallel vector field $\hat{\mathcal{Z}} \in \mathfrak{X}(\hat{N})$ under a (local) conformal diffeomorphism $\Psi: N^{m+1} \rightarrow \hat{N}^{m+1}$. If $f: M^{m} \rightarrow N^{n}$ has arbitrary codimension, the two properties are related as follows.

Corollary 4. Let $N^{n}$ be a Riemannian manifold and let $\mathcal{Z} \in \mathfrak{X}(N)$ be $\Psi$-related to a parallel vector field $\hat{\mathcal{Z}} \in \mathfrak{X}(\hat{N})$ under a (local) conformal diffeomorphism $\Psi: N^{n} \rightarrow \hat{N}^{n}$. Then an isometric immersion $f: M^{m} \rightarrow N^{n}$ has the principal direction property with respect to $\mathcal{Z}$ if and only if $\mathcal{Z}_{f}^{T}$ is nowhere vanishing, the ratio $\left\|\mathcal{Z}_{f}^{\perp}\right\| /\left\|\mathcal{Z}_{f}^{T}\right\|$ is constant along $\left\{\mathcal{Z}_{f}^{T}\right\}^{\perp}$ and either $\mathcal{Z}_{f}^{\perp}$ is everywhere vanishing or $\zeta_{f}=\mathcal{Z}_{f}^{\perp} /\left\|\mathcal{Z}_{f}^{\perp}\right\|$ is parallel in the normal connection along $\left\{\mathcal{Z}_{f}^{T}\right\}^{\perp}$.

Proof. Let $\hat{f}=\Psi \circ f: M^{m} \rightarrow \hat{N}^{n}$. By the first assertion in Lemma 2, $\hat{f}$ has the principal direction property with respect to $\hat{\mathcal{Z}}$ if and only if $\hat{\mathcal{Z}}_{f}^{T}$ is nowhere vanishing and $\hat{\mathcal{Z}}_{\hat{f}}^{\perp}$ is parallel in the normal connection along $\left\{\hat{\mathcal{Z}}_{\hat{f}}^{T}\right\}^{\perp}$. The latter condition is equivalent to $\left\|\hat{\mathcal{Z}}_{\hat{f}}^{\perp}\right\|$ being constant along $\left\{\hat{\mathcal{Z}}_{\hat{f}}^{T}\right\}^{\perp}$ and, if this constant is nonzero, to the unit vector field $\hat{\mathcal{Z}}_{\hat{\hat{f}}}^{\perp} /\left\|\hat{\mathcal{Z}}_{\hat{f}}^{\perp}\right\|$ being parallel in the normal connection along $\left\{\hat{\mathcal{Z}}_{\hat{f}}^{T}\right\}^{\perp}$. Since $\hat{\mathcal{Z}}$ has constant length, $\left\|\hat{\mathcal{Z}}_{\hat{f}}^{\perp}\right\|$ being a nonzero constant along $\left\{\hat{\mathcal{Z}}_{\hat{f}}^{T}\right\}^{\perp}$ is equivalent to the ratio $\left\|\hat{\mathcal{Z}}_{\hat{f}}^{\perp}\right\| /\left\|\hat{\mathcal{Z}}_{\hat{f}}^{T}\right\|$ being constant along $\left\{\hat{\mathcal{Z}}_{\hat{f}}^{T}\right\}^{\perp}$. Now, since $\Psi: N^{n} \rightarrow \hat{N}^{n}$ is a (local) conformal diffeomorphism, $\hat{\nabla}_{X}^{\perp} \hat{\mathcal{Z}}_{\hat{f}}^{\perp}$ and $\nabla_{X}^{\perp} \mathcal{Z}_{f}^{\perp}$ are related by

$$
\begin{aligned}
\hat{\nabla}_{X}^{\perp} \hat{\mathcal{Z}}_{\hat{f}}^{\perp} & =\Psi_{*} \nabla_{X}^{\perp} \mathcal{Z}_{f}^{\perp}+\frac{1}{\varphi \circ f} X(\varphi \circ f) \Psi_{*} \mathcal{Z}_{f}^{\perp} \\
& =\Psi_{*} \nabla_{X}^{\perp} \mathcal{Z}_{f}^{\perp}+\frac{1}{\varphi \circ f} X(\varphi \circ f) \hat{\mathcal{Z}}_{\hat{f}}^{\perp}
\end{aligned}
$$

where, as above, $\varphi$ is the conformal factor of $\Psi$. Therefore, if $\hat{\mathcal{Z}}_{\hat{f}}^{\perp}$ is nowhere vanishing, then the unit vector field $\hat{\mathcal{Z}}_{\hat{f}}^{\perp} /\left\|\hat{\mathcal{Z}}_{\hat{f}}^{\perp}\right\|$ is parallel in the normal connection along $\left\{\hat{\mathcal{Z}}_{\hat{f}}^{T}\right\}^{\perp}$ if and only if the unit vector field $\mathcal{Z}_{f}^{\perp} /\left\|\mathcal{Z}_{f}^{\perp}\right\|$ is parallel in the normal connection along $\left\{\mathcal{Z}_{f}^{T}\right\}^{\perp}$. Moreover, by parts $(i)$ and $(i i)$ of Lemma 3 , if $\hat{\mathcal{Z}}_{\hat{f}}^{T}$ is nowhere vanishing, then the ratio $\left\|\hat{\mathcal{Z}}_{\hat{f}}^{\perp}\right\| /\left\|\hat{\mathcal{Z}}_{\hat{f}}^{T}\right\|$ is constant along $\left\{\hat{\mathcal{Z}}_{\hat{f}}^{T}\right\}^{\perp}$ if and only if the ratio $\left\|\mathcal{Z}_{f}^{\perp}\right\| /\left\|\mathcal{Z}_{f}^{T}\right\|$ is constant along $\left\{\mathcal{Z}_{f}^{T}\right\}^{\perp}$. 


\section{Constant ratio property with respect to $\frac{\partial}{\partial t}$}

In this section we investigate the isometric immersions $f: M^{m} \rightarrow \mathbb{Q}_{\epsilon}^{n} \times \mathbb{R}$, with $m \geq 2$, into the Riemannian product of $\mathbb{Q}_{\epsilon}^{n}$ and $\mathbb{R}$, that have the constant ratio property with respect to the unit vector field $\mathcal{Z}=\frac{\partial}{\partial t}$, tangent to the factor $\mathbb{R}$. We may assume that $\epsilon \in\{-1,0,1\}$, and we will use the following models for $\mathbb{Q}_{0}^{n}, \mathbb{Q}_{1}^{n}$ and $\mathbb{Q}_{-1}^{n}$, respectively:

$$
\begin{aligned}
& \mathbb{R}^{n}, \\
& \mathbb{S}^{n}=\left\{\left(x_{1}, \ldots, x_{n+1}\right) \in \mathbb{R}^{n+1}: x_{1}^{2}+\ldots+x_{n+1}^{2}=1\right\}, \\
& \mathbb{H}^{n}=\left\{\left(x_{1}, \ldots, x_{n+1}\right) \in \mathbb{R}_{1}^{n+1}: x_{1}^{2}+\ldots+x_{n}^{2}-x_{n+1}^{2}=-1, x_{n+1}>0\right\},
\end{aligned}
$$

which immediately give models for $\mathbb{Q}_{0}^{n} \times \mathbb{R}, \mathbb{Q}_{1}^{n} \times \mathbb{R}$ and $\mathbb{Q}_{-1}^{n} \times \mathbb{R}$ as subsets of $\mathbb{R}^{n+1}$, $\mathbb{R}^{n+2}$ and $\mathbb{R}_{1}^{n+2}$, respectively. In all cases, the last coordinate on the ambient space coincides with the coordinate on the factor $\mathbb{R}$ in $\mathbb{Q}_{\epsilon}^{n} \times \mathbb{R}$, which we always denote by $t$. In particular, the vector field $\frac{\partial}{\partial t}$ can be seen as a constant unit vector field on $\mathbb{R}^{n+1}$, $\mathbb{R}^{n+2}$ or $\mathbb{R}_{1}^{n+2}$, respectively, and for $\epsilon=0$ the problem at hand comes down to finding isometric immersions into $\mathbb{R}^{n+1}$ having the constant ratio property with respect to a constant vector field.

Note that, in all three cases, two classes of trivial examples of submanifolds of $\mathbb{Q}_{\epsilon}^{n} \times \mathbb{R}$ with the constant ratio property with respect to $\mathcal{Z}=\frac{\partial}{\partial t}$ occur. First, if $N^{m-1}$ is a submanifold of $\mathbb{Q}_{\epsilon}^{n}$, then any open subset of the product immersion $N^{m-1} \times \mathbb{R}$ into $\mathbb{Q}_{\epsilon}^{n} \times \mathbb{R}$ has everywhere vanishing normal vector field $\mathcal{Z}_{f}^{\perp}$. These examples are called vertical cylinders. On the other hand, any submanifold of a horizontal slice $\mathbb{Q}_{\epsilon}^{n} \times\left\{t_{0}\right\}$, where $t_{0} \in \mathbb{R}$ is fixed, has everywhere vanishing tangent vector field $\mathcal{Z}_{f}^{T}$. In the following we describe how any other example arises.

Let $M^{m}=J \times N^{m-1}$ be a product manifold endowed with a polar metric

$$
d \sigma^{2}=\pi_{1}^{*} d s^{2}+\pi_{2}^{*} g_{s},
$$

where $\pi_{1}: M^{m} \rightarrow J$ and $\pi_{2}: M^{m} \rightarrow N^{m-1}$ are the canonical projections, $d s^{2}$ is the standard metric on $J$ and $\left\{g_{s}\right\}_{s \in J}$ is a one-parameter family of metrics on $N^{m-1}$ indexed on $J$. That the metric of $M^{m}=J \times N^{m-1}$ is the polar metric (7) is equivalent to requiring the metric to be orthogonal and the curves $s \mapsto(s, x), x \in N^{m-1}$, to be geodesics in $M^{m}$ (see Proposition 2.3 of [20]). A particular case of a polar metric (7) is a warped product metric

$$
\pi_{1}^{*} d s^{2}+\left(\rho \circ \pi_{1}\right)^{2} \pi_{2}^{*} g
$$

for some $\rho \in C^{\infty}(J)$ and some fixed metric $g$ on $N^{m-1}$, which corresponds to the case in which all metrics $g_{s}, s \in J$, are homothetical to a fixed metric $g$ on $N^{m-1}$.

Theorem 5. Let $\phi: M^{m} \rightarrow \mathbb{Q}_{\epsilon}^{n} \subset \mathbb{R}_{(1)}^{n+1}, m \geq 2, \epsilon \in\{-1,0,1\}$, be an isometric immersion of a product manifold $M^{m}=I \times N^{m-1}$ endowed with a polar metric. Then the map $f: M^{m} \rightarrow \mathbb{Q}_{\epsilon}^{n} \times \mathbb{R}$ given by

$$
f(s, x)=(\phi(s, x), A s), \quad A \neq 0,
$$


is an immersion with the constant ratio property with respect to the unit vector field $\mathcal{Z}=\frac{\partial}{\partial t}$ tangent to the factor $\mathbb{R}$.

Conversely, if $f: M^{m} \rightarrow \mathbb{Q}_{\epsilon}^{n} \times \mathbb{R}, m \geq 2, \epsilon \in\{-1,0,1\}$, is an isometric immersion with the constant ratio property with respect to $\mathcal{Z}=\frac{\partial}{\partial t}$, then either $f(M)$ is an open part of a vertical cylinder, or it is contained in a horizontal slice, or $f$ is locally given as above (globally, with $J=\mathbb{R}$, if the integral curves of $\mathcal{Z}_{f}^{T}$ are defined on $\mathbb{R}$ ).

If $m=n$, then Theorem 5 reduces to Corollary 2 of [19], by taking into account the following elementary observation that we state as a lemma.

Lemma 6. If $\phi: M^{n} \rightarrow \mathbb{Q}_{\epsilon}^{n} \subset \mathbb{R}_{(1)}^{n+1}$, with $n \geq 2$ and $\epsilon \in\{-1,0,1\}$, is a local isometry of a product manifold $M^{n}=I \times N^{n-1}$ endowed with a polar metric, then there exists a hypersurface $\phi_{0}: N^{n-1} \rightarrow \mathbb{Q}_{\epsilon}^{n}$ such that $\phi_{s}:=\phi(s, \cdot): N^{n-1} \rightarrow \mathbb{Q}_{\epsilon}^{n}$ is the family of parallel hypersurfaces to $\phi_{0}$ indexed on $J$, that is,

$$
\phi_{s}(x)=C_{\epsilon}(s) \phi_{0}(x)+S_{\epsilon}(s) N(x)
$$

where $N$ is a unit normal vector field to $\phi_{0}$ and $\left(C_{\epsilon}(s), S_{\epsilon}(s)\right)$ is either $(\cos s, \sin s)$, $(1, s)$ or $(\cosh s, \sinh s)$, depending on whether $\epsilon=1,0$ or -1 , respectively.

Before giving the proof of Theorem 5 we show the following fact, which sheds light on how product manifolds $M^{m}=J \times N^{m-1}$ with a polar metric arise.

Lemma 7. Let $M^{m}$ be a Riemannian manifold and let $\rho \in C^{\infty}(M)$ be such that $\operatorname{grad} \rho$ has unit length. Then $M^{m}$ is locally isometric to a product manifold $J \times N^{m-1}$ endowed with a polar metric ( 7$)$, with the curves $s \mapsto\left(s, x_{0}\right), x_{0} \in N^{m-1}$, corresponding to the integral curves of grad $\rho$. The assertion holds globally, with $J=\mathbb{R}$, if the integral curves of $\operatorname{grad} \rho$ are defined on $\mathbb{R}$.

Proof. Denote $\mathcal{T}=\operatorname{grad} \rho$. Then $\left\langle\nabla_{\mathcal{T}} \mathcal{T}, X\right\rangle=\left\langle\nabla_{X} \mathcal{T}, \mathcal{T}\right\rangle=(1 / 2) X\langle\mathcal{T}, \mathcal{T}\rangle=0$ for all $X \in \mathfrak{X}(M)$, thus the integral curves of $\mathcal{T}$ are geodesics of $M^{m}$. On the other hand, since $\mathcal{T}$ is a gradient vetor field, then the distribution $\{\mathcal{T}\}^{\perp}$ is integrable, its leaves being the level sets of $\rho$. By Theorem 2.7 of [20], $M^{m}$ is locally isometric to a product $J \times N^{m-1}$ endowed with a polar metric (7). Moreover, also by that theorem, $M^{m}$ is globally isometric to a product $\mathbb{R} \times N^{m-1}$ endowed with such a polar metric if the integral curves of $\mathcal{T}$ are complete geodesics.

We point out that any Riemannian manifold $M^{m}$ admits locally a function $\rho$ whose gradient has unit length. For instance, if $x \in M^{m}$ and $U \subset T_{x} M$ is an open neighborhood of $0 \in T_{x} M$ restricted to which the exponential map $\exp _{x}$ is a diffeomorphism onto $V \subset M^{m}$, then the map $\rho: V \backslash\{x\} \rightarrow \mathbb{R}$ given by $\rho(y)=\left\|\exp _{x}^{-1} y\right\|$ has this property. The integral curves of $\operatorname{grad} \rho$ are radial geodesics issuing from $x$ and the leaves of the orthogonal distribution are the geodesic hyperspheres centered at $x$. 
Therefore, starting with any isometric immersion $f: M^{m} \rightarrow \mathbb{Q}_{\epsilon}^{n}, m \geq 2, \epsilon \in$ $\{-1,0,1\}$, one can produce an immersion $\phi: J \times N^{m-1} \rightarrow \mathbb{Q}_{\epsilon}^{n}$ of a product manifold $J \times N^{m-1}$, with $J \subset \mathbb{R}$ an open interval, whose induced metric is the polar metric (7). It suffices to compose a map $\psi: J \times N^{m-1} \rightarrow U$ onto an open subset $U$ of $M^{m}$ whose induced metric is such a polar metric, as the isometry in the preceding paragraph, with the restriction $\left.f\right|_{U}$ of $f$ to $U$.

Proof of Theorem 5: Since $\mathcal{Z}=\frac{\partial}{\partial t}$ has constant length, proving that $f$ has the constant ratio property with respect to $\mathcal{Z}$ is equivalent to showing that either $\mathcal{Z}_{f}^{T}$ or $\mathcal{Z}_{f}^{\perp}$ has constant length. From (8) we have

$$
0=X\langle f, \mathcal{Z}\rangle=\left\langle f_{*} X, \mathcal{Z}\right\rangle=\left\langle X, \mathcal{Z}_{f}^{T}\right\rangle_{f}
$$

for all $X \in \mathfrak{X}(N)$, where $\langle\cdot, \cdot\rangle_{f}$ denotes the metric $d \sigma^{2}=\left(1+A^{2}\right) \pi_{1}^{*} d s^{2}+\pi_{2}^{*} g_{s}$ induced by $f$. It follows that $\mathcal{Z}_{f}^{T}=\lambda \frac{\partial}{\partial s}$ for some $\lambda \in C^{\infty}(M)$. On the other hand,

$$
A=\frac{\partial}{\partial s}\langle f, \mathcal{Z}\rangle=\left\langle f_{*} \frac{\partial}{\partial s}, \mathcal{Z}\right\rangle=\left\langle\frac{\partial}{\partial s}, \mathcal{Z}_{f}^{T}\right\rangle_{f}=\lambda\left\langle\frac{\partial}{\partial s}, \frac{\partial}{\partial s}\right\rangle_{f}=\left(1+A^{2}\right) \lambda .
$$

Therefore $\mathcal{Z}_{f}^{T}$ has constant length $|A|\left(1+A^{2}\right)^{-1 / 2}$.

We now prove the converse. If either $\mathcal{Z}_{f}^{\perp}$ or $\mathcal{Z}_{f}^{T}$ vanishes identically, then $f(M)$ is an open part of a vertical cylinder or it is contained in a horizontal slice, respectively. Suppose from now on that neither of these possibilities occur. Denoting $F=\langle f, \mathcal{Z}\rangle$ we have

$$
X(F)=X\langle f, \mathcal{Z}\rangle=\left\langle f_{*} X, \mathcal{Z}\right\rangle=\left\langle X, \mathcal{Z}_{f}^{T}\right\rangle_{f}
$$

for all $X \in \mathfrak{X}(M)$. This means that $\mathcal{Z}_{f}^{T}$ is the gradient of $F$. Since $f$ has the constant ratio property with respect to $\mathcal{Z}=\frac{\partial}{\partial t}$, which has constant length, also $\mathcal{Z}_{f}^{T}$ has constant length. It follows from Lemma 7 that $M^{m}$ is locally isometric to a product $M^{m}=$ $I \times N^{m-1}$ endowed with a polar metric (7), and actually globally if the integral curves of $\mathcal{Z}_{f}^{T}$ are complete geodesics. Furthermore, from

$$
\operatorname{Hess} F\left(\mathcal{Z}_{f}^{T}, \mathcal{Z}_{f}^{T}\right)=\left\langle\nabla_{\mathcal{Z}_{f}^{T}} \mathcal{Z}_{f}^{T}, \mathcal{Z}_{f}^{T}\right\rangle_{f}=0
$$

it follows that $F$ depends linearly on $s$.

\subsection{The warped product case}

We now consider isometric immersions $f: M^{m} \rightarrow I \times{ }_{\rho} \mathbb{Q}_{\epsilon}^{n}$ into a warped product $I \times{ }_{\rho} \mathbb{Q}_{\epsilon}^{n}$, where $I \subset \mathbb{R}$ is an open interval, endowed with the warped product metric

$$
\langle,\rangle=\pi_{1}^{*} d t^{2}+\left(\rho \circ \pi_{1}\right)^{2} \pi_{2}^{*}\langle,\rangle_{\mathbb{Q}_{\epsilon}^{n}}
$$

that have the constant ratio property with respect to the unit vector field $\mathcal{Z}=\frac{\partial}{\partial t}$ tangent to the factor $I$. Here the warping function $\rho: I \rightarrow \mathbb{R}$ is any smooth positive function and $\pi_{1}: I \times \mathbb{Q}_{\epsilon}^{n} \rightarrow I, \pi_{2}: I \times \mathbb{Q}_{\epsilon}^{n} \rightarrow \mathbb{Q}_{\epsilon}^{n}$ denote the canonical projections. 
The classification of such isometric immersions will follow from part (iii) of Lemma 3 and Theorem 5 by remarking that $I \times{ }_{\rho} \mathbb{Q}_{\epsilon}^{n}$ is conformally diffeomorphic to an open subset of $\mathbb{Q}_{\epsilon}^{n} \times \mathbb{R}$. This observation goes back to Gerardus Mercator (1512-1594) (before calculus was invented!) in his construction of a conformal map of the sphere onto the plane from the standard parametrization of the sphere by the latitude and longitude. Namely, let $G: I \rightarrow \mathbb{R}$ be any primitive of the function $\frac{1}{\rho}: I \rightarrow \mathbb{R}$, that is, $G^{\prime}(t)=\frac{1}{\rho(t)}$ for all $t \in I$. Then $G$ is an increasing diffeomorphism onto an open interval $J \subset \mathbb{R}$, and the map $\phi: I \times{ }_{\rho} \mathbb{Q}_{\epsilon}^{n} \rightarrow \mathbb{Q}_{\epsilon}^{n} \times J \subset \mathbb{Q}_{\epsilon}^{n} \times \mathbb{R}$, defined as

$$
\phi(s, x)=(x, G(s)),
$$

is a conformal diffeomorphism between $I \times \times_{\rho} \mathbb{Q}_{\epsilon}^{n}$ and $\mathbb{Q}_{\epsilon}^{n} \times J$ with conformal factor $\lambda=\rho \circ \pi_{1}$, that is, its induced metric is $\lambda^{2}\langle$,$\rangle . Its inverse$

$$
\psi=\phi^{-1}: \mathbb{Q}_{\epsilon}^{n} \times J \rightarrow I \times{ }_{\rho} \mathbb{Q}_{\epsilon}^{n}
$$

is given by

$$
\psi(x, t)=(F(t), x),
$$

where $F=G^{-1}$, so that $F^{\prime}(t)=\rho(F(t))$ for all $t \in J$. Moreover,

$$
\Psi_{*}\left(x_{0}, t_{0}\right) \frac{\partial}{\partial t}\left(x_{0}, t_{0}\right)=F^{\prime}\left(t_{0}\right) \frac{\partial}{\partial t}\left(F\left(t_{0}\right), x_{0}\right)=\rho\left(F\left(t_{0}\right)\right) \frac{\partial}{\partial t}\left(F\left(t_{0}\right), x_{0}\right)
$$

for all $\left(x_{0}, t_{0}\right) \in \mathbb{Q}_{\epsilon}^{n} \times J$, where the former $\frac{\partial}{\partial t}$ denotes a unit tangent vector field on $J$, while the latter two denote a unit tangent vector field on $I$. Therefore, the following result is a consequence of part (iii) of Lemma 3 together with Theorem 5 .

Theorem 8. Let $I \times{ }_{\rho} \mathbb{Q}_{\epsilon}^{n}$ be a warped product of an open interval $I \subset \mathbb{R}$ and $\mathbb{Q}_{\epsilon}^{n}$, $\epsilon \in\{-1,0,1\}$. Let $F: \tilde{J} \rightarrow \tilde{I}$ be a diffeomorphism of an open interval $\tilde{J} \subset \mathbb{R}$ onto an open interval $\tilde{I} \subset I$ satisfying

$$
F^{\prime}(t)=\rho(F(t))
$$

for all $t \in \tilde{J}$, let $A \neq 0$ and let $J=h^{-1}(\tilde{J})$, where $h: \mathbb{R} \rightarrow \mathbb{R}$ is the linear map given by $h(t)=$ At for all $t \in \mathbb{R}$. Let $\phi: M^{m} \rightarrow \mathbb{Q}_{\epsilon}^{n}$ be an isometric immersion of the product manifold $M^{m}=J \times N^{m-1}, m \geq 2$, endowed with a polar metric (7). Then the map $f: M^{m} \rightarrow I \times{ }_{\rho} \mathbb{Q}_{\epsilon}^{n}$ given by

$$
f(s, x)=(F(A s), \phi(s, x))
$$

is an immersion with the constant ratio property with respect to the unit vector field $\mathcal{Z}=\frac{\partial}{\partial t}$ tangent to the factor $I$.

Conversely, if $f: M^{m} \rightarrow I \times_{\rho} \mathbb{Q}_{\epsilon}^{n}, m \geq 2, \epsilon \in\{-1,0,1\}$, is an isometric immersion that has the constant ratio property with respect to $\mathcal{Z}=\frac{\partial}{\partial t}$, then either $f(M)$ is an open part of a vertical cylinder $I \times{ }_{\rho} N^{m-1}$ or of a submanifold of a horizontal slice, or $f$ is locally given as above (globally, if the integral curves of $\mathcal{Z}_{f}^{T}$ are defined on $\mathbb{R}$ ).

In the case of hypersurfaces one has a more explicit description by taking Lemma 6 into account. 


\subsection{The space form case - loxodromic immersions}

There are well-known representations of any real space form $\mathbb{Q}_{\epsilon}^{n+1}, \epsilon \in\{1,0-1\}$ (or an open dense subset of it), as a warped product of an open interval and another space form, namely,

$$
\begin{array}{ll}
\mathbb{R}^{n+1} \backslash\{0\}=(0,+\infty) \times{ }_{\rho} \mathbb{S}^{n}, & \text { with } \rho(t)=t, \\
\mathbb{S}^{n+1} \backslash\{S, N\}=(0, \pi) \times{ }_{\rho} \mathbb{S}^{n}, & \text { with } \rho(t)=\sin t, \\
\mathbb{H}^{n+1} \backslash\{P\}=(0,+\infty) \times{ }_{\rho} \mathbb{S}^{n}, & \text { with } \rho(t)=\sinh t, \\
\mathbb{H}^{n+1}=\mathbb{R} \times{ }_{\rho} \mathbb{H}^{n}, & \text { with } \rho(t)=\cosh t, \\
\mathbb{H}^{n+1}=\mathbb{R} \times{ }_{\rho} \mathbb{R}^{n}, & \text { with } \rho(t)=e^{t} / \sqrt{2} .
\end{array}
$$

The first one is just the standard description of $\mathbb{R}^{n+1} \backslash\{0\}$ in spherical coordinates, given by the isometry

$$
(0, \infty) \times_{i d} \mathbb{S}^{n} \rightarrow \mathbb{R}^{n+1} \backslash\{0\}:(t, x) \mapsto t x
$$

The second one corresponds to the isometry of the unit sphere $\mathbb{S}^{n+1}$ minus the south and north poles $S=(0, \ldots, 0,-1)$ and $N=(0, \ldots, 0,1)$ given, with respect to the model (5) for $\mathbb{S}^{n}$ and the corresponding one for $\mathbb{S}^{n+1}$, by the map

$$
(0, \pi) \times \sin \mathbb{S}^{n} \rightarrow \mathbb{S}^{n+1} \backslash\{S, N\}:(t, x) \mapsto(\sin t) x+(\cos t) N .
$$

In terms of the model (5) for $\mathbb{S}^{n}$ and the one corresponding to (6) for $\mathbb{H}^{n+1}$, the representation (14) of $\mathbb{H}^{n+1}$ minus $P=(0, \ldots, 0,1)$ is given by the isometry

$$
(0,+\infty) \times_{\sinh } \mathbb{S}^{n} \rightarrow \mathbb{H}^{n+1} \backslash\{P\}:(t, x) \mapsto(\sinh t) x+(\cosh t) P
$$

For the representation (15) of $\mathbb{H}^{n+1}$, take the isometry

$$
\mathbb{R} \times_{\cosh } \mathbb{H}^{n} \rightarrow \mathbb{H}^{n+1}:(t, x) \mapsto(\sinh t) e+(\cosh t) x
$$

with $\mathbb{H}^{n} \subset \mathbb{R}_{1}^{n+1}=\{e\}^{\perp} \subset \mathbb{R}_{1}^{n+2}$, whereas for (16) the isometry is given by

$$
\mathbb{R} \times_{(1 / \sqrt{2}) \exp } \mathbb{R}^{n} \rightarrow \mathbb{H}^{n+1}:(t, x) \mapsto(1 / \sqrt{2}) e^{t}\left(v+x-(1 / 2)\|x\|^{2} w\right)-(1 / \sqrt{2}) e^{-t} w
$$

where $\{v, w\}$ is a pseudo-orthonormal basis of $\left(\mathbb{R}^{n}\right)^{\perp} \subset \mathbb{R}_{1}^{n+2}$, that is, $\langle v, v\rangle=0=\langle w, w\rangle$ and $\langle v, w\rangle=1$.

Classicaly, a unit speed curve $\gamma: I \rightarrow \mathbb{S}^{2}$ is called a loxodrome if its tangent vector makes a constant angle with the meridians of $\mathbb{S}^{2}$. One can similarly define a loxodrome $\gamma: I \rightarrow \mathbb{S}^{n+1}$ in $\mathbb{S}^{n+1}$ for any $n \geq 2$. Using the model (13) to describe $\mathbb{S}^{n+1}$, loxodromes in $\mathbb{S}^{n+1}$ are precisely the images under the isometry in (18) of the curves into $(0, \pi) \times_{\sin } \mathbb{S}^{n}$ that have the constant ratio property with respect to the unit tangent vector $\mathcal{Z}=\frac{\partial}{\partial t}$ to $(0, \pi)$. 
Let us denote by $\phi$ either of the isometries given by (17) to (21), and by $\frac{\partial}{\partial t}$ a unit vector field tangent to the one-dimensional factor of the corresponding warped-product space. Then the vector field $\phi_{*} \frac{\partial}{\partial t}$ in $\mathbb{Q}_{\epsilon}^{n+1}$ gives rise to a "radial" vector field $\mathcal{R}$ in $\mathbb{Q}_{\epsilon}^{n+1}$ (of three different types for $\epsilon=-1$ ). We will also refer to an isometric immersion into any of the models (12)-(16) having the constant ratio property with respect to the radial vector field $\mathcal{R}$ as a loxodromic isometric immersion. To distinguish the three possible cases in $\mathbb{H}^{n+1}$, we will call a loxodromic isometric immersion $f: M^{m} \rightarrow \mathbb{H}^{n+1}$ elliptic, hyperbolic or parabolic, depending on whether the radial vector field $\mathcal{R}$ is determined by an isometry $\phi$ as in (14), (15) or (16), respectively.

For $\epsilon=0$, the vector field $\mathcal{R}$ is the standard radial vector field, and a better approach to studying the constant ratio property with respect to that vector field will be given in Section 5. Therefore, we will omit this case here.

A description of all loxodromic isometric immersions $f: M^{m} \rightarrow \mathbb{Q}_{\epsilon}^{n+1}$ for $\epsilon \in\{-1,1\}$ follows immediately from Theorem 8. Namely, any loxodromic isometric immersion $f: M^{m} \rightarrow \mathbb{Q}_{\epsilon}^{n+1}$ is given by $f=\phi \circ \hat{f}$, where $\phi$ is one of the isometries given by (18) to (21) and $\hat{f}: M^{n} \rightarrow I \times{ }_{\rho} \mathbb{Q}_{\epsilon}^{n}$ is given by $(11)$, with $I=(0, \pi),(0, \infty)$ or $\mathbb{R}$, and $F: \tilde{J} \rightarrow \tilde{I}$ a diffeomorphism of an open interval $\tilde{J} \subset \mathbb{R}$ onto an open interval $\tilde{I} \subset I$ satisfying (10) for the restriction to $\tilde{I}$ of the corresponding warping function $\rho \in C^{\infty}(I)$ given as in either of equations (13) to (16).

For instance, any solution of the ODE $F^{\prime}(t)=\sin (F(t))$ on a maximal interval is given by $F: \mathbb{R} \rightarrow \mathbb{R}: t \mapsto 2 \arctan \left(e^{t-c}\right)$ for an arbitrary real constant $c$, which we can assume to be zero after a translation of the parameter $t$. Therefore, any loxodromic isometric immersion into $\mathbb{S}^{n+1}$ is given by a map $f: M^{m}:=J \times N^{m-1} \rightarrow \mathbb{S}^{n+1}$ defined by

$$
f(s, x)=\sin \left(2 \arctan \left(e^{(\sin \theta) s}\right)\right) \phi(s, x)+\cos \left(2 \arctan \left(e^{(\sin \theta) s}\right)\right) N
$$

where $\phi: M^{m} \rightarrow \mathbb{S}^{n}, m \geq 2$, is an isometric immersion of the product manifold $M^{m}=$ $J \times N^{m-1}$ endowed with the polar metric (7).

Note that, although Theorem 8 is formulated for $m \geq 2$, we can still interpret it for $m=1$, i.e., to describe constant ratio curves. In this case, the manifold $N^{m-1}$ collapses to a point and $M^{1}=J$ is an open interval, so that the preceding equation reduces to

$$
\begin{aligned}
f(s, x)= & \sin \left(2 \arctan \left(e^{(\sin \theta) s}\right)\right)(\cos ((\cos \theta) s), \sin ((\cos \theta) s), 0) \\
& +\cos \left(2 \arctan \left(e^{(\sin \theta) s}\right)\right)(0,0,1),
\end{aligned}
$$

which, under the reparametrizaton $u=2 \arctan \left(e^{(\sin \theta) s}\right)$, corresponds to the usual description of a loxodrome on $\mathbb{S}^{2} \subset \mathbb{R}^{3}$, namely,

$$
\alpha(u)=\left(\cos \left(\cot \theta \ln \left(\tan \frac{u}{2}\right)\right) \sin u, \sin \left(\cot \theta \ln \left(\tan \frac{u}{2}\right)\right) \sin u, \cos u\right) .
$$

To obtain the parabolic loxodromic isometric immersions into $\mathbb{H}^{n+1}$, first notice that any solution of the $\mathrm{ODE} F^{\prime}(t)=\sqrt{2} e^{F(t)}$ on a maximal interval is given by

$$
F:(-\infty, c / \sqrt{2}) \rightarrow \mathbb{R}: t \mapsto \log \frac{1}{c-\sqrt{2} t}
$$


for an arbitrary real constant $c$. Therefore, any parabolic loxodromic isometric immersion into $\mathbb{H}^{n+1}$ is given by a map $f: M^{m}:=J \times N^{m-1} \rightarrow \mathbb{H}^{n+1} \subset \mathbb{R}_{1}^{n+2}$ defined by

$$
\sqrt{2} f(s, x)=\frac{1}{c-\sqrt{2}(\sin \theta) s}\left(v+\phi(s, x)+\frac{1}{2}\|\phi(s, x)\|^{2} w\right)-(c-\sqrt{2}(\sin \theta) s) w
$$

where $\{v, w\}$ is a pseudo-orthonormal basis of $\left(\mathbb{R}^{n}\right)^{\perp} \subset \mathbb{R}_{1}^{n+2}$, with $\langle v, v\rangle=0=\langle w, w\rangle$ and $\langle v, w\rangle=1$, and $\phi: M^{m} \rightarrow \mathbb{R}^{n}, m \geq 2$, is an isometric immersion of the product manifold $M^{m}=J \times N^{m-1}$ endowed with the polar metric (7).

Elliptic and hyperbolic loxodromic isometric immersions into $\mathbb{H}^{n+1}$ can be explicitly computed in a similar way.

\section{Principal direction property with respect to $\frac{\partial}{\partial t}$}

Isometric immersions $f: M^{m} \rightarrow \mathbb{Q}_{\epsilon}^{n} \times \mathbb{R}$, with $m \geq 2$ and $\epsilon \in\{-1,0,1\}$ that satisfy the principal direction property with respect to $\mathcal{Z}=\frac{\partial}{\partial t}$ and which are not vertical cylinders were completely described in [19] for the case of hypersurfaces and in [15] for arbitrary codimension. The class of such isometric immersions was called class $\mathcal{A}$ in [15]. We will consider the case $\epsilon \in\{-1,1\}$, the case $\epsilon=0$ being similar.

Let $\phi: N^{m-1} \rightarrow \mathbb{Q}_{\epsilon}^{n}$ be an isometric immersion and assume that there exists an orthonormal set of normal vector fields $\xi_{1}, \ldots, \xi_{k}$ along $\phi$ that are parallel in the normal bundle. This assumption is satisfied, for instance, if $\phi$ has flat normal bundle. After including $\mathbb{Q}_{\epsilon}^{n}$ into $\mathbb{Q}_{\epsilon}^{n} \times \mathbb{R}$ in the standard way and using the models (5) and (6), we can look at $\phi$ as an immersion into $\mathbb{R}_{(1)}^{n+2}$ and at the vector fields $\xi_{1}, \ldots, \xi_{k}$ as vector fields on $\mathbb{R}_{(1)}^{n+2}$ along this immersion. Now add the following two vector fields along $\phi$ to this list:

$$
\xi_{k+1}(x)=\phi(x), \quad \xi_{k+2}(x)=\frac{\partial}{\partial t}(\phi(x))=(0, \ldots, 0,1)
$$

for all $x \in N^{m-1}$. Observe that $\xi_{1}, \ldots, \xi_{k+1}$ all have last component zero. The vector subbundle $E$ of the normal bundle of $\phi: N^{m-1} \rightarrow \mathbb{R}_{(1)}^{n+2}$, whose fiber $E(x)$ at $x \in N^{m-1}$ is spanned by $\xi_{1}(x), \ldots, \xi_{k+2}(x)$, is parallel and flat and we may define a vector bundle isometry

$$
\Xi: N^{m-1} \times \mathbb{R}_{(1)}^{k+2} \rightarrow E
$$

by

$$
\Xi\left(x,\left(y_{1}, \ldots, y_{k+2}\right)\right)=\sum_{i=1}^{k+2} y_{i} \xi_{i}(x) .
$$

Now, let $\gamma: I \rightarrow \mathbb{Q}_{\epsilon}^{k} \times \mathbb{R} \subset \mathbb{R}_{(1)}^{k+2}, \gamma=\left(\gamma_{1}, \ldots, \gamma_{k+1}, \gamma_{k+2}\right)$, be a smooth regular curve such that $\gamma_{1}^{2}+\ldots+\gamma_{k}^{2}+\epsilon \gamma_{k+1}^{2}=\epsilon$ and $\gamma_{k+2}$ has non-vanishing derivative, and define a 
$\operatorname{map} f: N^{m-1} \times I \rightarrow \mathbb{Q}_{\epsilon}^{n} \times \mathbb{R}$ by

$$
\begin{aligned}
f(x, s) & =\Xi(x, \gamma(s))=\sum_{i=1}^{k+2} \gamma_{i}(s) \xi_{i}(x) \\
& =\sum_{i=1}^{k} \gamma_{i}(s) \xi_{i}(x)+\gamma_{k+1}(s) \phi(x)+\left(0, \ldots, 0, \gamma_{k+2}(s)\right)
\end{aligned}
$$

Notice that, if $\gamma$ parametrizes a line $\left\{p_{0}\right\} \times \mathbb{R}$ in $\mathbb{Q}_{\epsilon}^{k} \times \mathbb{R} \subset \mathbb{E}^{k+2}$, then the map $f$ parametrizes a vertical cylinder. Geometrically, the image of $f$ is generated by parallel transporting the curve $\gamma$ in a product submanifold $\mathbb{Q}_{\epsilon}^{k} \times \mathbb{R}$ of a fixed normal space of $\phi$ in $\mathbb{R}_{(1)}^{n+2}$ with respect to its normal connection.

The following result from [15], which we reformulate a bit for our purposes, states that the above construction gives all submanifolds of class $\mathcal{A}$, that is, all submanifolds in $\mathbb{Q}_{\epsilon}^{n} \times \mathbb{R}$ that have the principal direction property with respect to $\mathcal{Z}=\frac{\partial}{\partial t}$.

Theorem 9 ( [15]). The restriction of the map $f: N^{m-1} \times I \rightarrow \mathbb{Q}_{\epsilon}^{n} \times \mathbb{R}$ given by (22) to the subset of its regular points is an immersion with the principal direction property with respect to $\mathcal{Z}=\frac{\partial}{\partial t}$.

Conversely, if $f: M^{m} \rightarrow \mathbb{Q}_{\epsilon}^{n} \times \mathbb{R}, m \geq 2$, is an isometric immersion with the principal direction property with respect to $\mathcal{Z}=\frac{\bar{\partial}}{\partial t}$, then it is locally given in this way (globally, if the integral curves of $\mathcal{Z}_{f}^{T}$ are defined on $\mathbb{R}$ ).

The next result classifies isometric immersions $f: M^{m} \rightarrow \mathbb{Q}_{\epsilon}^{n} \times \mathbb{R}$ with both the principal direction and constant ratio properties with respect to $\mathcal{Z}=\frac{\partial}{\partial t}$.

Corollary 10. Let $f: M^{m}=N^{m-1} \times I \rightarrow \mathbb{Q}_{\epsilon}^{n} \times \mathbb{R}$ be given by (22) for a regular curve $\gamma: I \subset \mathbb{R} \rightarrow \mathbb{Q}_{\epsilon}^{k} \times \mathbb{R} \subset \mathbb{R}_{(1)}^{k+2}, \gamma(s)=\left(\gamma_{1}(s), \ldots, \gamma_{k+1}(s), \gamma_{k+2}(s)\right)$, such that $\bar{\gamma}: I \rightarrow \mathbb{Q}_{\epsilon}^{k} \subset \mathbb{R}_{(1)}^{k+1}, \bar{\gamma}(s)=\left(\gamma_{1}(s), \ldots, \gamma_{k+1}(s)\right)$, is a unit-speed curve and $\left.\gamma_{k+2}(s)\right)=$ As for some $A \neq 0$. Then the restriction of $f$ to the subset of its regular points is an immersion with both the principal direction and constant ratio properties with respect to $\mathcal{Z}=\frac{\partial}{\partial t}$.

Conversely, any isometric immersion $f: M^{m} \rightarrow \mathbb{Q}_{\epsilon}^{n} \times \mathbb{R}, m \geq 2$, with both the principal direction and constant ratio properties with respect to $\mathcal{Z}=\frac{\partial}{\partial t}$, is locally given in this way (globally, if the integral curves of $\mathcal{Z}_{f}^{T}$ are defined on $\mathbb{R}$ ).

Proof. Let $f: M^{m}=N^{m-1} \times I \rightarrow \mathbb{Q}_{\epsilon}^{n} \times \mathbb{R} \subset \mathbb{R}_{(1)}^{n+2}$ be the map given by (22) for a regular curve $\gamma: I \subset \mathbb{R} \rightarrow \mathbb{Q}_{\epsilon}^{k} \times \mathbb{R} \subset \mathbb{R}_{(1)}^{k+2}, \gamma(s)=\left(\gamma_{1}(s), \ldots, \gamma_{k+1}(s), \gamma_{k+2}(s)\right)$. By [15, Proposition 3.2], the normal space $N_{f} M(x, s)$ of $f$ in $\mathbb{R}_{(1)}^{n+2}$ at $(x, s)$ is given by

$$
N_{f} M(x, s)=\Xi\left(x, \operatorname{span}\left\{\gamma^{\prime}(s)\right\}^{\perp}\right) \subset N_{\phi} N(x),
$$


and the shape operator of $f$ with respect to $\Xi(x, \zeta)$ satisfies

$$
A_{\Xi(x, \zeta)}^{f}(x, s) \frac{\partial}{\partial s}=\frac{\left\langle\gamma^{\prime \prime}(s), \zeta\right\rangle}{\left\langle\gamma^{\prime}(s), \gamma^{\prime}(s)\right\rangle} \frac{\partial}{\partial s}
$$

for all $\zeta \in \mathbb{R}_{(1)}^{k+2}$ with $\left\langle\zeta, \gamma^{\prime}(s)\right\rangle=0$. On the other hand, we have

$$
\mathcal{Z}_{f}^{\perp}=\Xi\left(x, e_{k+2}^{\perp}\right)
$$

where $e_{k+2}^{\perp}=e_{k+2}-\left\langle\gamma^{\prime}(s), \gamma^{\prime}(s)\right\rangle^{-1}\left\langle e_{k+2}, \gamma^{\prime}(s)\right\rangle \gamma^{\prime}(s)$ is the normal component of $e_{k+2}$ along $\gamma$. It follows that $\left\langle A_{\mathcal{Z}_{f}} \mathcal{Z}_{f}^{T}, \mathcal{Z}_{f}^{T}\right\rangle=0$ if and only if $\left\langle\gamma^{\prime \prime}(s), e_{k+2}\right\rangle=0$ for all $s \in I$, that is, if and only if $\gamma_{k+2}$ is linear.

By Lemma 2, special cases of isometric immersions $f: M^{m} \rightarrow \mathbb{Q}_{\epsilon}^{n} \times \mathbb{R}$ with both the principal direction and constant ratio properties with respect to $\mathcal{Z}=\frac{\partial}{\partial t}$ are those for which $\mathcal{Z}_{f}^{\perp}$ is parallel in the normal connection. These isometric immersions were shown in $[15]$ to be precisely the ones given by (22) with $\gamma: I \rightarrow \mathbb{Q}_{\epsilon}^{k} \times \mathbb{R}$ a geodesic of $\mathbb{Q}_{\epsilon}^{k} \times \mathbb{R}$.

In the case of hypersurfaces, Theorem 9 reduces to Theorem 1 in [19] by taking Lemma 6 into account.

\subsection{The warped product case}

The invariance of the principal direction property with respect to conformal changes of the ambient metric (see part ( $i v$ ) of Lemma 3) can be used to determine the isometric immersions $f: M^{m} \rightarrow I \times{ }_{\rho} \mathbb{Q}_{\epsilon}^{n}$ into a warped product $I \times_{\rho} \mathbb{Q}_{\epsilon}^{n}$ that have the principal direction property with respect to the unit vector field $\mathcal{Z}=\frac{\partial}{\partial t}$ tangent to the factor $I$.

Namely, let $\psi: \mathbb{Q}_{\epsilon}^{n} \times J \rightarrow I \times \times_{\rho} \mathbb{Q}_{\epsilon}^{n}$ be the conformal diffeomorphism given by (9). Then, by part $(i v)$ of Lemma 3 and Theorem 9 , any such isometric immersion is given by $f=\psi \circ \hat{f}$, where $\hat{f}: M^{m} \rightarrow \mathbb{Q}_{\epsilon}^{n} \times \mathbb{R}$ is the restriction of the map $\hat{f}: N^{m-1} \times I \rightarrow$ $\mathbb{Q}_{\epsilon}^{n} \times \mathbb{R}$ given by $(22)$ to the subset $M^{m} \subset N^{m-1} \times I$ of its regular points, endowed with the induced metric, with the smooth regular curve $\gamma: I \rightarrow \mathbb{Q}_{\epsilon}^{k} \times \mathbb{R} \subset \mathbb{R}_{(1)}^{k+2}$, $\gamma=\left(\gamma_{1}, \ldots, \gamma_{k+1}, \gamma_{k+2}\right)$, satisfying $\gamma(I) \subset \mathbb{Q}_{\epsilon}^{n} \times J$, that is, $\gamma_{k+2}(I) \subset J$. Moreover, choosing $\hat{f}$ as in Corollary 10, the isometric immersions $f=\psi \circ \hat{f}$ give all isometric immersions into $I \times_{\rho} \mathbb{Q}_{\epsilon}^{n}$ that have both the constant ratio and principal direction properties with respect to $\mathcal{Z}=\frac{\partial}{\partial t}$.

In a similar way one can describe all isometric immersions $\tilde{f}: M^{m} \rightarrow \mathbb{Q}_{\epsilon}^{n}$ that have the principal direction property (or both the constant ratio and principal direction properties) with respect to a radial vector field $\mathcal{R}$ as in Section 3.2. It suffices to compose the isometric immersions produced as described in the previous paragraph with one of the isometries $\phi$ given by (18) to (21). 


\section{Constant ratio property for radial vector fields}

In this section and the next, we study Euclidean submanifolds with the constant ratio or the principal direction property with respect to a radial vector field on $\mathbb{R}^{n} \backslash\{0\}$. In view of the remark after Definition 1, without loss of generality we may work with the position vector field given by $\mathcal{R}(y)=y$ for all $y \in \mathbb{R}^{n} \backslash\{0\}$.

Our results will follow by combining the assertions in parts (iii) and (iv) of Lemma 3 with the results of Sections 3 and 4, making use of the fact that the map

$$
\Psi: \mathbb{S}^{n-1} \times \mathbb{R} \rightarrow \mathbb{R}^{n} \backslash\{0\}:(x, t) \mapsto e^{t} x
$$

is a conformal diffeomorphism, with inverse

$$
\Phi=\Psi^{-1}: \mathbb{R}^{n} \backslash\{0\} \rightarrow \mathbb{S}^{n-1} \times \mathbb{R}: y \mapsto\left(\frac{y}{\|y\|}, \log \|y\|\right)
$$

Indeed, it is easily checked that the conformal factors of $\Psi$ and $\Phi$ are, respectively, $\varphi(x, t)=e^{t}$ and $\phi(y)=\|y\|^{-1}$. Moreover, the unit vector field $\frac{\partial}{\partial t} \in \mathfrak{X}\left(\mathbb{S}^{n-1} \times \mathbb{R}\right)$ tangent to the factor $\mathbb{R}$ and the radial vector field $\mathcal{R} \in \mathfrak{X}\left(\mathbb{R}^{n} \backslash\{0\}\right)$ are $\Psi$-related, that is, for all $(x, t) \in \mathbb{S}^{n-1} \times \mathbb{R}$ we have

$$
\Psi_{*}(x, t) \frac{\partial}{\partial t}(x, t)=\mathcal{R}(\Psi(x, t)) .
$$

A description of the submanifolds of $\mathbb{R}^{n} \backslash\{0\}$ having the constant ratio property with respect to $\mathcal{R}$ was given in [2]. An alternative description is as follows.

Theorem 11. Let $\phi: M^{m} \rightarrow \mathbb{S}^{n-1}, m \geq 2$, be an isometric immersion of a product manifold $M^{m}=J \times N^{m-1}$, with $J \subset \mathbb{R}$ an open interval, endowed with the polar metric (7). Then the map $f: M^{m} \rightarrow \mathbb{R}^{n} \backslash\{0\}$ given by

$$
f(s, x)=e^{A s} \phi(s, x), \quad A \neq 0,
$$

defines an immersion with the constant ratio property with respect to the radial vector field $\mathcal{R}$.

Conversely, if $f: M^{m} \rightarrow \mathbb{R}^{n} \backslash\{0\}$ is an isometric immersion with the constant ratio property with respect to $\mathcal{R}$, then either $f(M)$ is contained in a hypersphere centered at the origin, or $f(M)$ is an open subset of a cone over a submanifold $N^{m-1}$ of $\mathbb{S}^{n-1}$, or $f$ is given locally as above (globally, if the integral curves of $\mathcal{R}_{f}^{T}$ are defined on $\mathbb{R}$ ).

Proof. By part (iii) of Lemma 3, an isometric immersion $f: M^{m} \rightarrow \mathbb{R}^{n} \backslash\{0\}$ has the constant ratio property with respect to $\mathcal{R}$ if and only if $f=\Psi \circ \hat{f}$ for some isometric immersion $\hat{f}: M^{m} \rightarrow \mathbb{S}^{n-1} \times \mathbb{R}$ that has the constant ratio property with respect to $\frac{\partial}{\partial t}$, where $\Psi$ is given by (24). The statement then follows from Theorem 5 . Notice that $f(M)$ being contained in a hypersphere centered at the origin is equivalent to $\hat{f}(M)$ 
being contained in a horizontal slice of $\mathbb{S}^{n-1} \times \mathbb{R}$, whereas $f(M)$ being an open subset of a cone over a submanifold $N^{m-1}$ of $\mathbb{S}^{n-1}$ is equivalent to $\hat{f}(M)$ being an open subset of a vertical cylinder $\hat{N}^{m-1} \times \mathbb{R}$.

Notice that the cases in which $f(M)$ is contained in a hypersphere centered at the origin or is an open subset of a cone over a submanifold $N^{m-1}$ of $\mathbb{S}^{n-1}$ correspond, respectively, to the cases in which $\mathcal{R}_{f}^{T}$ or $\mathcal{R}_{f}^{\perp}$ vanishes identically.

Euclidean hypersurfaces of any dimension $n$ that have the constant ratio property with respect to the radial vector field $\mathcal{R}$ have been described in [1] (an alternative description was given in [16] for $n=2$ and in [21] for arbitrary $n$ ). The next consequence of Theorem 11 and Lemma 6 is essentially the description in [21].

Corollary 12. Let $\phi_{0}: N^{n-2} \rightarrow \mathbb{S}^{n-1} \subset \mathbb{R}^{n}$ be a hypersurface and let $\phi_{s}: N^{n-2} \rightarrow \mathbb{S}^{n-1}$ be the family of parallel hypersurfaces to $\phi_{0}$, indexed on the open interval $J \subset \mathbb{R}$. Then the map $f: M^{n-1}:=N^{n-2} \times J \rightarrow \mathbb{R}^{n} \backslash\{0\}$, given by

$$
f(x, s)=e^{A s} \phi_{s}(x), \quad A \neq 0,
$$

defines, at regular points, a hypersurface that has the constant ratio property with respect to the radial vector field $\mathcal{R}$.

Conversely, if $f: M^{n-1} \rightarrow \mathbb{R}^{n}$ is a hypersurface with the constant ratio property with respect to the radial vector field $\mathcal{R}$, then either $f(M)$ is an open subset of a hypersphere centered at the origin or of a cone over a hypersurface of $\mathbb{S}^{n-1}$, or $f$ is locally given as above.

Notice that the $s$-coordinate curves of the hypersurfaces given by (26) are logarithmic spirals, the plane curves that have the constant ratio property with respect to the radial vector field $\mathcal{R}$ on $\mathbb{R}^{2} \backslash\{0\}$.

\section{$5.1 \quad T$-constant and $N$-constant Euclidean submanifolds}

Related classes of isometric immersions $f: M^{m} \rightarrow \mathbb{R}^{n}$ are those for which either $\left\|\mathcal{R}_{f}^{T}\right\|$ or $\left\|\mathcal{R}_{f}^{\perp}\right\|$ is constant on $M^{m}$. These were called $T$-submanifolds and $N$-submanifolds, respectively, in [3], where a description of them was given. An alternative description of those submanifolds can be derived as follows with our methods.

Theorem 13. Let $\phi: M^{m} \rightarrow \mathbb{S}^{n-1}, m \geq 2$, be an isometric immersion of a product manifold $M^{m}=J \times N^{m-1}$, with $J \subset \mathbb{R}$ an open interval, endowed with the polar metric (7). Define $f: M^{m} \rightarrow \mathbb{R}^{n} \backslash\{0\}$ by

$$
f(s, x)=\rho(s) \phi(s, x)
$$

with

$$
\rho(s)=\sec (s+C), \quad C \in \mathbb{R},
$$


(respectively,

$$
\rho(s)=\sqrt{1+G^{2}(s+C)}, \quad C \in \mathbb{R},
$$

where $G: \mathbb{R} \rightarrow \mathbb{R}$ is the inverse function of $F: \mathbb{R} \rightarrow \mathbb{R}, F(x)=x-\arctan x)$. Then the restriction of $f$ to the subset of its regular points defines an immersion such that $\left\|\mathcal{R}_{f}^{\perp}\right\|$ (respectively, $\left\|\mathcal{R}_{f}^{T}\right\|$ ) has unit length on $M^{m}$.

Conversely, if $f: M^{m} \rightarrow \mathbb{R}^{n} \backslash\{0\}$ is an isometric immersion such that $\left\|\mathcal{R}_{f}^{\perp}\right\|$ (respectively, $\left.\left\|\mathcal{R}_{f}^{T}\right\|\right)$ has a constant value $K$ on $M^{m}$, then either $f(M)$ is an open subset of a cone over a submanifold of $\mathbb{S}^{n-1}$ (respectively, $f(M)$ is contained in a hypersphere centered at the origin) if $K=0$, or, if otherwise, $f$ is locally (globally, if the integral curves of $\mathcal{R}_{f}^{T}$ are defined on $\mathbb{R}$ ) the composition of a map as above with a homothety of $\mathbb{R}^{n}$ of ratio $K\left(X \in \mathbb{R}^{n} \mapsto K X\right)$.

First we prove the following lemma.

Lemma 14. Let $f: M^{m} \rightarrow \mathbb{R}^{n} \backslash\{0\}$ be an isometric immersion and let $\hat{f}=\Phi \circ f$, where $\Phi: \mathbb{R}^{n} \backslash \mathbb{R} \rightarrow \mathbb{S}^{n-1} \times \mathbb{R}$ is the conformal diffeomorphism given by (25). Then

$$
\left\|\mathcal{R}_{f}^{\perp}\right\|_{f}=e^{h}\left\|\hat{\mathcal{Z}}_{\hat{f}}^{\perp}\right\|_{\hat{f}} \text { and }\left\|\mathcal{R}_{f}^{T}\right\|_{f}=e^{h}\left\|\hat{\mathcal{Z}}_{\hat{f}}^{T}\right\|_{\hat{f}},
$$

where $\hat{\mathcal{Z}}=\frac{\partial}{\partial t}$ and $h=\langle\hat{f}, \hat{\mathcal{Z}}\rangle$ is the height function of $\hat{f}$ with respect to $\hat{\mathcal{Z}}$. Moreover, the assertions

(i) $\left\|\mathcal{R}_{f}^{\perp}\right\|$ is constant on $M^{m}$;

(ii) $\nabla_{\hat{\mathcal{Z}}_{\hat{f}}^{T}} \hat{\mathcal{Z}}_{\hat{f}}^{T}=\left(1-\left\|\hat{\mathcal{Z}}_{\hat{f}}^{T}\right\|^{2}\right) \hat{\mathcal{Z}}_{\hat{f}}^{T}$;

(iii) $A_{\hat{\mathcal{Z}}_{\hat{f}} \hat{\mathcal{Z}}_{\hat{f}}^{T}}^{\hat{\mathcal{f}}^{T}}=\left(1-\left\|\hat{\mathcal{Z}}_{\hat{f}}^{T}\right\|^{2}\right) \hat{\mathcal{Z}}_{\hat{f}}^{T}$

are equivalent, and the same holds for the assertions

(i') $\left\|\mathcal{R}_{f}^{T}\right\|$ is constant on $M^{m}$;

(ii') $\nabla_{\hat{\mathcal{Z}}_{\hat{f}}^{T}} \hat{\mathcal{Z}}_{\hat{f}}^{T}=-\left\|\hat{\mathcal{Z}}_{\hat{f}}^{T}\right\|^{2} \hat{\mathcal{Z}}_{\hat{f}}^{T}$.

Proof. For any $x \in M^{m}$ we have

$$
\begin{aligned}
\left\langle\hat{\mathcal{Z}}_{\hat{f}}^{\perp}(x), \hat{\mathcal{Z}}_{\hat{f}}^{\perp}(x)\right\rangle & =\left\langle\Phi_{*}(f(x)) \mathcal{R}_{f}^{\perp}(x), \Phi_{*}(f(x)) \mathcal{R}_{f}^{\perp}(x)\right\rangle \\
& =\phi^{2}(f(x))\left\langle\mathcal{R}_{f}^{\perp}(x), \mathcal{R}_{f}^{\perp}(x)\right\rangle \\
& =\frac{\left\langle\mathcal{R}_{f}^{\perp}(x), \mathcal{R}_{f}^{\perp}(x)\right\rangle}{\|f(x)\|^{2}} \\
& =e^{-2\langle\hat{f}(x), \hat{\mathcal{Z}}(\hat{f}(x))\rangle}\left\langle\mathcal{R}_{f}^{\perp}(x), \mathcal{R}_{f}^{\perp}(x)\right\rangle
\end{aligned}
$$


and

$$
\begin{aligned}
\left\langle\hat{\mathcal{Z}}_{\hat{f}}^{T}(x), \hat{\mathcal{Z}}_{\hat{f}}^{T}(x)\right\rangle_{\hat{f}} & =\phi^{2}(f(x))\left\langle\mathcal{R}_{f}^{T}(x), \mathcal{R}_{f}^{T}(x)\right\rangle_{f} \\
& =\frac{\left\langle\mathcal{R}_{f}^{T}(x), \mathcal{R}_{f}^{T}(x)\right\rangle_{f}}{\|f(x)\|^{2}} \\
& =e^{-2\langle\hat{f}(x), \hat{\mathcal{Z}}(\hat{f}(x))\rangle}\left\langle\mathcal{R}_{f}^{T}(x), \mathcal{R}_{f}^{T}(x)\right\rangle_{f} .
\end{aligned}
$$

By the first formula in (29), $\left\|\mathcal{R}_{f}^{\perp}\right\|$ is constant on $M^{m}$, say, $\left\|\mathcal{R}_{f}^{\perp}(x)\right\|=K \in \mathbb{R}$ for all $x \in M^{m}$, if and only if

$$
\left\langle\hat{\mathcal{Z}}_{\hat{f}}^{\perp}(x), \hat{\mathcal{Z}}_{\hat{f}}^{\perp}(x)\right\rangle=K^{2} e^{-2\langle\hat{f}(x), \hat{\mathcal{Z}}(\hat{f}(x))\rangle}
$$

for all $x \in M^{m}$. Differentiating with respect to $X \in \mathfrak{X}(M)$ implies that this is equivalent to

$$
\left\langle\nabla_{X}^{\perp} \hat{\mathcal{Z}}_{\hat{f}}^{\perp}, \hat{\mathcal{Z}}_{\hat{f}}^{\perp}\right\rangle=-\left\langle\hat{\mathcal{Z}}_{\hat{f}}^{\perp}, \hat{\mathcal{Z}}_{\hat{f}}^{\perp}\right\rangle\left\langle\hat{\mathcal{Z}}_{\hat{f}}^{T}, X\right\rangle
$$

for all $X \in \mathfrak{X}(M)$. Using (2), the preceding equation can be written as that in item (iii), which by (1) is equivalent to the formula in item $(i i)$.

Finally, by the second formula in (29), $\left\|\mathcal{R}_{f}^{T}\right\|$ is constant on $M^{m}$, say, $\left\|\mathcal{R}_{f}^{T}(x)\right\|=$ $K \in \mathbb{R}$ for all $x \in M^{m}$, if and only if

$$
\left\langle\hat{\mathcal{Z}}_{\hat{f}}^{T}(x), \hat{\mathcal{Z}}_{\hat{f}}^{T}(x)\right\rangle=K^{2} e^{-2\langle\hat{f}(x), \hat{\mathcal{Z}}(\hat{f}(x))\rangle}
$$

for all $x \in M^{m}$. Differentiating with respect to $X \in \mathfrak{X}(M)$ implies that this is equivalent to

$$
\left\langle\nabla_{X} \hat{\mathcal{Z}}_{\hat{f}}^{T}, \hat{\mathcal{Z}}_{\hat{f}}^{T}\right\rangle=-\left\langle\hat{\mathcal{Z}}_{\hat{f}}^{T}, \hat{\mathcal{Z}}_{\hat{f}}^{T}\right\rangle\left\langle\hat{\mathcal{Z}}_{\hat{f}}^{T}, X\right\rangle
$$

for all $X \in \mathfrak{X}(M)$. Using that $\hat{\mathcal{Z}}_{\hat{f}}^{T}$ is a gradient vector field implies that the preceding equation is equivalent to that in item $\left(i i^{\prime}\right)$.

Proof of Theorem 13: Let $\hat{f}=\Phi \circ f$, where $\Phi: \mathbb{R}^{n} \backslash\{0\} \rightarrow \mathbb{S}^{n-1} \times \mathbb{R}$ is the conformal diffeomorphism given by (25). Then

$$
\hat{f}(s, x)=(\phi(s, x), h(s))
$$

for all $(s, x) \in M^{m}$. Arguing as in the beginning of the proof of Theorem 5 , we see that, if $\hat{\mathcal{Z}}=\frac{\partial}{\partial t} \in \mathfrak{X}\left(\mathbb{S}^{n-1} \times \mathbb{R}\right)$, then $\hat{\mathcal{Z}}_{\hat{f}}^{T}=\lambda \frac{\partial}{\partial s}$ for some $\lambda \in C^{\infty}(M)$. Moreover,

$$
h^{\prime}(s)=\frac{\partial}{\partial s}\langle\hat{f}, \hat{\mathcal{Z}}\rangle=\left\langle\hat{f}_{*} \frac{\partial}{\partial s}, \hat{\mathcal{Z}}\right\rangle=\left\langle\frac{\partial}{\partial s}, \hat{\mathcal{Z}}_{\hat{f}}^{T}\right\rangle_{\hat{f}}=\lambda\left\langle\frac{\partial}{\partial s}, \frac{\partial}{\partial s}\right\rangle_{\hat{f}}=\left(1+\left(h^{\prime}(s)\right)^{2}\right) \lambda
$$


hence

$$
\left\|\hat{\mathcal{Z}}_{\hat{f}}^{T}\right\|^{2}=\frac{\left(h^{\prime}(s)\right)^{2}}{1+\left(h^{\prime}(s)\right)^{2}} \text { and }\left\|\hat{\mathcal{Z}}_{\hat{f}}^{\perp}\right\|^{2}=\frac{1}{1+\left(h^{\prime}(s)\right)^{2}},
$$

which implies that $e^{h}\left\|\hat{\mathcal{Z}}_{\hat{\hat{f}}}^{\perp}\right\|$ (respectively, $e^{h}\left\|\hat{\mathcal{Z}}_{\hat{f}}^{T}\right\|$ ) is constant if $h$ is given by (27) (respectively, (28)). Thus the statement follows from (29).

Now we prove the converse. If either $\left\|\mathcal{R}_{f}^{\perp}\right\|$ or $\left\|\mathcal{R}_{f}^{T}\right\|$ is constant on $M^{m}$, then both the equations in items $(i i)$ and $\left(i i^{\prime}\right)$ imply that the integral curves of $\hat{\mathcal{Z}}_{\hat{f}}^{T} /\left\|\hat{\mathcal{Z}}_{\hat{f}}^{T}\right\|$ are geodesics. Moreover, using that $\hat{\mathcal{Z}}_{\hat{f}}^{T}$ is the gradient of the height function $h=\langle\hat{f}, \hat{\mathcal{Z}}\rangle$, and hence that the orthogonal distribution $\hat{\mathcal{Z}}_{\hat{f}}^{T}$ is integrable, we see that $M^{m}$ is locally diffeomorphic to a product manifold $M^{m}=I \times N^{m-1}$, and that

$$
f(x, s)=(\phi(x, s), h(s)
$$

for some immersion $\phi: M^{m} \rightarrow \mathbb{S}^{m-1}$ and some $h \in C^{\infty}(I)$. Moreover, if for each $s \in I$ we denote by $g_{s}$ the metric induced by the map

$$
x \in M^{m} \mapsto \phi(x, s) \in \mathbb{S}^{m-1},
$$

then the fact that the $s$-coordinate curves $s \mapsto(s, x)$ (the integral curves of $\hat{\mathcal{Z}}_{\hat{f}}^{T}$ ) are reparametrizations of geodesics says that the metric induced by $f$ is

$$
d \tilde{\sigma}=\left(1+\left(h^{\prime}(s)\right)^{2}\right) d s^{2}+g_{s} .
$$

Therefore, as in the proof of the direct statement, the assumption that $\left\|\mathcal{R}_{f}^{\perp}\right\|$ or $\left\|\mathcal{R}_{f}^{T}\right\|$ has a constant value $K$ on $M^{m}$ translates, respectively, into the ODEs

$$
\frac{e^{2 h}}{1+\left(h^{\prime}\right)^{2}}=K \text { and } \frac{e^{2 h}\left(h^{\prime}\right)^{2}}{1+\left(h^{\prime}\right)^{2}}=K
$$

for $h$, whose solutions are easily checked to be given by (27) and (28), respectively.

\section{Principal direction property for radial vector fields}

Corollary 4 takes the following simpler form for radial vector fields.

Corollary 15. An isometric immersion $f: M^{m} \rightarrow \mathbb{R}^{n} \backslash\{0\}$ has the principal direction property with respect to the radial vector field $\mathcal{R}$ if and only if $\mathcal{R}_{f}^{\perp}$ is parallel along $\left\{\mathcal{R}_{f}^{T}\right\}^{\perp}$ with respect to the normal connection.

Proof. Denoting by $\tilde{\nabla}$ the Euclidean connection and by $\nabla$ the Levi-Civita connection of $M^{n}$, the Gauss and Weingarten formulas yield

$$
\begin{aligned}
f_{*} X & =\tilde{\nabla}_{X}(\mathcal{R} \circ f)=\tilde{\nabla}_{X}\left(f_{*} \mathcal{R}_{f}^{T}+\mathcal{R}_{f}^{\perp}\right) \\
& =f_{*} \nabla_{X} \mathcal{R}_{f}^{T}+\alpha_{f}\left(X, \mathcal{R}_{f}^{T}\right)-f_{*} A_{\mathcal{R}_{f}^{\perp}} X+\nabla_{X}^{\perp} \mathcal{R}_{f}^{\perp} .
\end{aligned}
$$


The normal component of this equation reads $\nabla_{X}^{\perp} \mathcal{R}_{f}^{\perp}=-\alpha_{f}\left(X, \mathcal{R}_{f}^{T}\right)$, which implies the statement.

In the case of hypersurfaces, Corollary 15 reads as follows.

Corollary 16. A hypersurface $f: M^{n-1} \rightarrow \mathbb{R}^{n} \backslash\{0\}$ has the principal direction property with respect to the radial vector field $\mathcal{R}$ if and only if $\left\|\mathcal{R}_{f}^{\perp}\right\|$ is constant along $\left\{\mathcal{R}_{f}^{T}\right\}^{\perp}$.

By the observation after Lemma 3, every hypersurface of $\mathbb{R}^{n} \backslash\{0\}$ that has the constant ratio property with respect to $\mathcal{R}$ also has the principal direction property with respect to $\mathcal{R}$. Indeed, the radial vector field $\mathcal{R}$ is $\Psi$-related to the parallel unit vector field $\frac{\partial}{\partial t} \in \mathfrak{X}\left(\mathbb{S}^{n-1} \times \mathbb{R}\right)$ tangent to the factor $\mathbb{R}$, where $\Psi: \mathbb{S}^{n-1} \times \mathbb{R} \rightarrow \mathbb{R}^{n} \backslash\{0\}$ is the conformal diffeomorphism given by (24). This also follows from the preceding corollary by noticing that, since $\|\mathcal{R} \circ f\|$ is constant along $\left\{\mathcal{R}_{f}^{T}\right\}^{\perp}$, then $\left\|\mathcal{R}_{f}^{\perp}\right\|$ is also constant along $\left\{\mathcal{R}_{f}^{T}\right\}^{\perp}$ if $f$ is a hypersurface with the constant ratio property with respect to $\mathcal{R}$.

The next result gives a description of all isometric immersions $f: M^{m} \rightarrow \mathbb{R}^{n} \backslash\{0\}$, $m \geq 2$, that have the principal direction property with respect to $\mathcal{R}$.

Theorem 17. Let $\phi: N^{m-1} \rightarrow \mathbb{S}^{n-1} \subset \mathbb{R}^{n}$ be an isometric immersion along which there exists an orthonormal set of normal vector fields $\xi_{1}, \ldots, \xi_{k}$ that are parallel in the normal bundle. Let $\gamma: I \rightarrow \mathbb{S}^{k} \times \mathbb{R} \subset \mathbb{R}^{k+2}$, with $\gamma(s)=\left(\gamma_{1}(s), \ldots, \gamma_{k+1}(s), \gamma_{k+2}(s)\right)$, be a smooth regular curve such that $\gamma_{k+2}$ has non-vanishing derivative. Then the restriction of the map $f: N^{m-1} \times I \rightarrow \mathbb{R}^{n} \backslash\{0\}$, given by

$$
f(x, s)=e^{\gamma_{k+2}(s)}\left(\sum_{i=1}^{k} \gamma_{i}(s) \xi_{i}(x)+\gamma_{k+1}(s) \phi(x)\right),
$$

to the subset of its regular points defines an immersion with the principal direction property with respect to $\mathcal{R}$.

Conversely, if $f: M^{m} \rightarrow \mathbb{R}^{n} \backslash\{0\}$ is an isometric immersion with the principal direction property with respect to $\mathcal{R}$, then either $f(M)$ is an open subset of a cone over a submanifold $N^{m-1}$ of $\mathbb{S}^{n-1}$, or $f$ is given locally as above (globally, if the geodesic integral curves of $\mathcal{R}_{f}^{T}$ are defined on $\mathbb{R}$ ).

Proof. By part (iv) of Lemma 3, an isometric immersion $f: M^{m} \rightarrow \mathbb{R}^{n} \backslash\{0\}$ has the principal direction property with respect to $\mathcal{R}$ if and only if $f=\Psi \circ \hat{f}$ for some isometric immersion $\hat{f}: M^{m} \rightarrow \mathbb{S}^{n-1} \times \mathbb{R}$ with the principal direction property with respect to $\frac{\partial}{\partial t}$. The statement then follows from Theorem 9 .

Corollary 18. Let $\phi_{0}: N^{n-2} \rightarrow \mathbb{S}^{n-1} \subset \mathbb{R}^{n}$ be a hypersurface and let $\phi_{s}: N^{n-2} \rightarrow \mathbb{S}^{n-1}$ be the family of parallel hypersurfaces to $\phi_{0}$, indexed on an open interval $J \subset \mathbb{R}$. Then the restriction of the map $f: M^{n-1}:=N^{n-2} \times J \rightarrow \mathbb{R}^{n} \backslash\{0\}$, given by

$$
f(x, s)=e^{a(s)} \phi_{s}(x),
$$


to the subset of its regular points defines a hypersurface with the principal direction property with respect to $\mathcal{R}$.

Conversely, if $f: M^{n-1} \rightarrow \mathbb{R}^{n} \backslash\{0\}, n \geq 3$, is an isometric immersion with the principal direction property with respect to $\mathcal{R}$, then either $f(M)$ is an open subset of a cone over a hypersurface of $\mathbb{S}^{n-1}$, or $f$ is given locally as above (globally, if the integral curves of $\mathcal{R}_{f}^{T}$ are defined on $\left.\mathbb{R}\right)$.

Notice that $f(M)$ is foliated by the plane spirals

$$
s \mapsto f(x, s)=e^{a(s)}(\cos s \phi(x)+\sin s N(x)),
$$

and the orthogonal hypersurfaces $x \mapsto f(x, s)$ are homothetical to the parallel hypersurface $\phi_{s}$ of $\phi$.

For $n=3$, Corollary 18 reduces to the main theorem of [17], where surfaces in $\mathbb{R}^{3} \backslash\{0\}$ with the principal direction property with respect to $\mathcal{R}$ were called generalized constant ratio surfaces, in view of the fact that any constant ratio surface in $\mathbb{R}^{3}$ has this property, as pointed out after Corollary 16. This terminology is not appropriate for Euclidean submanifolds of codimension greater than one, for in this case the constant ratio property with respect to $\mathcal{R}$ no longer implies the principal direction property with respect to $\mathcal{R}$.

The following result classifies isometric immersions into $\mathbb{R}^{n} \backslash\{0\}$ that have both the constant ratio and the principal direction properties with respect to $\mathcal{R}$.

Corollary 19. Let $f: M^{m}=N^{m-1} \times I \rightarrow \mathbb{R}^{n} \backslash\{0\}$ be given by (30) for a regular curve $\gamma: I \subset \mathbb{R} \rightarrow \mathbb{S}^{k} \times \mathbb{R}, \gamma(s)=\left(\gamma_{1}(s), \ldots, \gamma_{k+1}(s), \gamma_{k+2}(s)\right)$, such that $\bar{\gamma}: I \rightarrow \mathbb{S}^{k} \subset \mathbb{R}^{k+1}$, $\bar{\gamma}(s)=\left(\gamma_{1}(s), \ldots, \gamma_{k+1}(s)\right)$, is a unit-speed curve and $\left.\gamma_{k+2}(s)\right)=$ As for some $A \neq 0$. Then the restriction of $f$ to the subset of its regular points is an immersion with both the principal direction and constant ratio properties with respect to $\mathcal{R}$.

Conversely, if $f: M^{m} \rightarrow \mathbb{R}^{n} \backslash\{0\}, m \geq 2$, is an isometric immersion with both the constant ratio and principal direction properties with respect to $\mathcal{R}$, then either $f(M)$ is an open subset of a cone over a submanifold $N^{m-1}$ of $\mathbb{S}^{n-1}$, or $f$ is given locally as above (globally, if the integral curves of $\mathcal{R}_{f}^{T}$ are defined on $\mathbb{R}$ ).

Proof. By parts (iii) and (iv) of Lemma 3, any isometric immersion $f: M^{m} \rightarrow \mathbb{R}^{n} \backslash\{0\}$ that has both the constant ratio and principal direction properties with respect to $\mathcal{R}$ is given by $f=\Psi \circ \hat{f}$ for some isometric immersion $\hat{f}: M^{m} \rightarrow \mathbb{S}^{n-1} \times \mathbb{R}$ that has both the constant ratio and principal direction properties with respect to $\frac{\partial}{\partial t}$, where $\Psi$ is given by (24). The statement then follows from Corollary 10.

\subsection{Euclidean submanifolds with parallel $\mathcal{R}_{f}^{\perp}$}

In view of Corollary 15, it is natural to ask which isometric immersions $f: M^{m} \rightarrow$ $\mathbb{R}^{n} \backslash\{0\}$ have the property that $\mathcal{R}_{f}^{\perp}$ is parallel on $M^{m}$ with respect to the normal connection. The next result classifies such submanifolds. 
Theorem 20. Let $f: M^{m}=N^{m-1} \times I \rightarrow \mathbb{R}^{n} \backslash\{0\}$ be given by (30) for a regular curve $\gamma: I \subset \mathbb{R} \rightarrow \mathbb{S}^{k} \times \mathbb{R}, \gamma(s)=\left(\gamma_{1}(s), \ldots, \gamma_{k+1}(s), \gamma_{k+2}(s)\right)$, such that $\bar{\gamma}: I \rightarrow \mathbb{S}^{k} \subset \mathbb{R}^{k+1}$, $\bar{\gamma}(s)=\left(\gamma_{1}(s), \ldots, \gamma_{k+1}(s)\right)$, is a geodesic and $\gamma_{k+2}(s)=\log (\sec (s+C))$ for some $C \in \mathbb{R}$ and all $s \in I$. Then the restriction of $f$ to the subset of its regular points is an immersion with the property that $\mathcal{R}_{f}^{\perp}$ is parallel in the normal connection.

Conversely, if $f: M^{m} \rightarrow \mathbb{R}^{n} \backslash\{0\}$ is an isometric immersion with the property that $\mathcal{R}_{f}^{\perp}$ is parallel in the normal connection, then either $f(M)$ is an open subset of a cone over a submanifold $N^{m-1}$ of $\mathbb{S}^{n-1}$, or $f$ is given locally as above (globally, if the integral curves of $\mathcal{R}_{f}^{T}$ are defined on $\left.\mathbb{R}\right)$, up to a homothety of $\mathbb{R}^{n}$.

First we prove the following lemma

Lemma 21. Let $f: M^{m} \rightarrow \mathbb{R}^{n} \backslash\{0\}$ be an isometric immersion and let $\hat{f}=\Phi \circ f$, where $\Phi: \mathbb{R}^{n} \backslash\{0\} \rightarrow \mathbb{S}^{n-1} \times \mathbb{R}$ is the conformal diffeomorphism given by (25). Then $\mathcal{R}_{f}^{\perp}$ is parallel on $M^{m}$ with respect to the normal connection if and only if

$$
A_{\xi}^{\hat{f}} \hat{\mathcal{Z}}_{\hat{f}}^{T}=\left\langle\hat{\mathcal{Z}}_{\hat{f}}^{\perp}, \xi\right\rangle \hat{\mathcal{Z}}_{\hat{f}}^{T}
$$

for all $\xi \in \Gamma\left(N_{\hat{f}} M\right)$, where $\hat{\mathcal{Z}}=\frac{\partial}{\partial t}$.

Proof. By (3), the normal derivatives $\hat{\nabla}_{X}^{\perp} \hat{\mathcal{Z}}_{\hat{f}}^{\perp}$ and $\nabla_{X}^{\perp} \mathcal{R} \frac{\perp}{f}$ are related by

$$
\hat{\nabla}_{X}^{\perp} \hat{\mathcal{Z}}_{\hat{f}}^{\perp}=\Phi_{*} \nabla_{X}^{\perp} \mathcal{R}_{f}^{\perp}+\frac{X(\phi \circ f)}{\phi \circ f} \hat{\mathcal{Z}}_{\hat{f}}^{\perp}
$$

for any $X \in \mathfrak{X}(M)$, where $\phi$ is the conformal factor of $\Phi$. Thus $\mathcal{R}_{f}^{\perp}$ is parallel on $M^{m}$ with respect to the normal connection if and only if

$$
\hat{\nabla}_{X}^{\perp} \hat{\mathcal{Z}}_{\hat{f}}^{\perp}=\frac{X(\phi \circ f)}{\phi \circ f} \hat{\mathcal{Z}}_{\hat{f}}^{\perp}
$$

for all $X \in \mathfrak{X}(M)$. Now, $\phi(y)=\frac{1}{\|y\|}$ for all $y \in \mathbb{R}^{n} \backslash\{0\}$, so

$$
\begin{aligned}
\frac{X(\phi \circ f)}{\phi \circ f} & =-X(\log \|f\|) \\
& =-X\langle\Phi \circ f, \hat{\mathcal{Z}}\rangle \\
& =-X\langle\hat{f}, \hat{\mathcal{Z}}\rangle \\
& =-\left\langle X, \hat{\mathcal{Z}}_{\hat{f}}^{T}\right\rangle
\end{aligned}
$$

for all $X \in \mathfrak{X}(M)$, and therefore (32) becomes

$$
\hat{\nabla}_{X}^{\perp} \hat{\mathcal{Z}}_{\hat{f}}^{\perp}=-\left\langle X, \hat{\mathcal{Z}}_{\hat{f}}^{T}\right\rangle \hat{\mathcal{Z}}_{\hat{f}}^{\perp}
$$

for all $X \in \mathfrak{X}(M)$. By (2), the preceding equation is equivalent to (31). 
Proof of Theorem 20: Let $\hat{f}=\Phi \circ f$, where $\Phi: \mathbb{R}^{n} \backslash\{0\} \rightarrow \mathbb{S}^{n-1} \times \mathbb{R}$ is the conformal diffeomorphism given by (25). Then $\hat{f}$ is given by $(22)$ in terms of a regular curve $\gamma: I \subset \mathbb{R} \rightarrow \mathbb{S}^{k} \times \mathbb{R} \subset \mathbb{R}^{k+2}, \gamma(s)=\left(\gamma_{1}(s), \ldots, \gamma_{k+1}(s), \gamma_{k+2}(s)\right)$. Thus $\hat{f}$ has the principal direction property with respect to $\hat{\mathcal{Z}}=\frac{\partial}{\partial t}$ by Theorem 9. Moreover, since $\bar{\gamma}: I \rightarrow \mathbb{S}^{k} \subset \mathbb{R}^{k+1}, \bar{\gamma}(s)=\left(\gamma_{1}(s), \ldots, \gamma_{k+1}(s)\right)$, is a geodesic of $\mathbb{S}^{k}$, it follows from (23) that $A_{\xi}^{\hat{f}} \hat{\mathcal{Z}}_{\hat{f}}^{T}=0$ for all $\xi \in \Gamma\left(N_{\hat{f}} M\right)$ with $\left\langle\xi, \hat{\mathcal{Z}}_{\hat{\hat{f}}}^{\perp}\right\rangle=0$. Finally, since the height function $\langle\hat{f}, \hat{\mathcal{Z}}\rangle=\gamma_{k+2}(s)=\log (\sec (s+C))$ for some $C \in \mathbb{R}$ and all $s \in I$, it follows from Theorem 13 that $\mathcal{R}_{f}^{\perp}$ has constant length. By item (iii) of Lemma 14, formula (31) also holds for $\xi=\hat{\mathcal{Z}} \frac{\perp}{\hat{f}}$.

Conversely, if $f: M^{m} \rightarrow \mathbb{R}^{n} \backslash\{0\}$ is an isometric immersion with the property that $\mathcal{R}_{f}^{\perp}$ is parallel in the normal connection, then $f$ has the principal direction property with respect to $\mathcal{R}$ by Corollary 15 . Therefore $\hat{f}=\Phi \circ f$, where $\Phi: \mathbb{R}^{n} \backslash\{0\} \rightarrow \mathbb{S}^{n-1} \times \mathbb{R}$ is the conformal diffeomorphism given by (25), has the principal direction property with respect to $\hat{\mathcal{Z}}=\frac{\partial}{\partial t}$ by part (iv) of Lemma 3 , and hence it is given by (22) in terms of a regular curve $\gamma: I \subset \mathbb{R} \rightarrow \mathbb{S}^{k} \times \mathbb{R} \subset \mathbb{R}^{k+2}, \gamma(s)=\left(\gamma_{1}(s), \ldots, \gamma_{k+1}(s), \gamma_{k+2}(s)\right)$, such that $\gamma_{k+2}^{\prime}$ has nonvanishing derivative by Theorem 9 . Since $A_{\xi}^{\hat{f}} \hat{\mathcal{Z}}_{\hat{f}}^{T}=0$ for all $\xi \in \Gamma\left(N_{\hat{f}} M\right)$ with $\left\langle\xi, \hat{\mathcal{Z}}_{\hat{f}}^{\perp}\right\rangle=0$ by (31), it follows from (23) that $\bar{\gamma}: I \rightarrow \mathbb{S}^{k} \subset \mathbb{R}^{k+1}$, $\bar{\gamma}(s)=\left(\gamma_{1}(s), \ldots, \gamma_{k+1}(s)\right)$, is a geodesic of $\mathbb{S}^{k}$. Finally, since $\mathcal{R}_{f}^{\perp}$ has constant length, it follows from Theorem 13 that $\gamma_{k+2}(s)=\log (K \sec (s+C))$ for some $K>0, C \in \mathbb{R}$ and all $s \in I$.

\section{The case of Killing vector fields}

Let $x_{1}, \ldots, x_{n+1}$ be the standard coordinates in $\mathbb{R}^{n+1}$ and let $\partial_{x_{i}}$ denote a unit vector field tangent to the $x_{i}$-coordinate curve, $1 \leq i \leq n+1$. The Lie algebra of Killing vector fields in $\mathbb{R}^{n+1}$ has dimension $\frac{1}{2}(n+1)(n+2)$ and is generated by the constant vector fields

$$
\partial_{x_{i}}, \quad 1 \leq i \leq n+1
$$

and the vector fields

$$
\mathcal{K}_{i j}=x_{i} \partial_{x_{j}}-x_{j} \partial_{x_{i}}, \quad 1 \leq i \neq j \leq n+1
$$

generating rotations around the linear subspaces $\mathbb{R}^{n-1}$ of $\mathbb{R}^{n+1}$ given by $x_{i}=0=x_{j}$.

In this section we describe all isometric immersions that have either the constant ratio or the principal direction property with respect to $\mathcal{K}_{i j}$. We work with, say, $\mathcal{K}_{n, n+1}$, which we denote simply by $\mathcal{K}$.

For this purpose, we make use of the conformal diffeomorphism between $\mathbb{H}^{n} \times \mathbb{S}^{1}$ and $\mathbb{R}^{n+1} \backslash \mathbb{R}^{n-1}$ given as follows. Let $e_{0}, e_{1}, \ldots, e_{n-1}, e_{n}$ be a pseudo-orthonormal basis 
of the Lorentzian space $\mathbb{R}_{1}^{n+1}$ satisfying

$$
\left\langle e_{0}, e_{0}\right\rangle=0=\left\langle e_{n}, e_{n}\right\rangle, \quad\left\langle e_{0}, e_{n}\right\rangle=-1 / 2 \text { and }\left\langle e_{i}, e_{j}\right\rangle=\delta_{i j}, \quad 1 \leq i \leq n-1, \quad 0 \leq j \leq n \text {. }
$$

Then the map $\Psi: \mathbb{H}^{n} \times \mathbb{S}^{1} \subset \mathbb{R}_{1}^{n+1} \times \mathbb{R}^{2} \rightarrow \mathbb{R}^{n+1} \backslash \mathbb{R}^{n-1}$ given by

$$
\Psi\left(x_{0} e_{0}+\ldots+x_{n} e_{n},\left(y_{1}, y_{2}\right)\right)=\frac{1}{x_{0}}\left(x_{1}, \ldots, x_{n-1}, y_{1}, y_{2}\right)
$$

is a conformal diffeomorphism with conformal factor

$$
\varphi\left(x_{0} e_{0}+\ldots+x_{n} e_{n},\left(y_{1}, y_{2}\right)\right)=\frac{1}{x_{0}},
$$

whose inverse $\Psi^{-1}: \mathbb{R}^{n+1} \backslash \mathbb{R}^{n-1} \rightarrow \mathbb{H}^{n} \times \mathbb{S}^{1} \subset \mathbb{R}_{1}^{n+1} \times \mathbb{R}^{2}$ is given by

$$
\Psi^{-1}\left(y_{1}, \ldots, y_{n+1}\right)=\frac{1}{\sqrt{y_{n}^{2}+y_{n+1}^{2}}}\left(e_{0}+\sum_{i=1}^{n-1} y_{i} e_{i}+\left(\sum_{i=1}^{n+1} y_{i}^{2}\right) e_{n},\left(y_{n}, y_{n+1}\right)\right) \text {. }
$$

Notice that the metric induced by the restriction of $\Psi^{-1}$ to each half-space of a hyperplane of $\mathbb{R}^{n+1}$ containing $\mathbb{R}^{n-1}$ is the standard hyperbolic metric of the half-space model of $\mathbb{H}^{n}$. In other words, the restriction of the conformal diffeomorphism $\Psi: \mathbb{H}^{n} \times \mathbb{S}^{1} \subset$ $\mathbb{R}_{1}^{n+1} \times \mathbb{R}^{2} \rightarrow \mathbb{R}^{n+1} \backslash \mathbb{R}^{n-1}$ to each slice $\mathbb{H}^{n} \times\{z\} \subset \mathbb{H}^{n} \times \mathbb{S}^{1}$ gives an isometry of the hyperboloidal model of $\mathbb{H}^{n}$ onto its half-space model.

Composing $\Psi$ with the isometric covering map

$$
\pi: \mathbb{H}^{n} \times \mathbb{R} \rightarrow \mathbb{H}^{n} \times \mathbb{S}^{1}:(x, t) \mapsto(x,(\cos t, \sin t))
$$

produces a conformal covering map $\tilde{\Psi}: \mathbb{H}^{n} \times \mathbb{R} \rightarrow \mathbb{R}^{n+1} \backslash \mathbb{R}^{n-1}$ given by

$$
\tilde{\Psi}\left(x_{0} e_{0}+\ldots+x_{n} e_{n}, t\right)=\frac{1}{x_{0}}\left(x_{1}, \ldots, x_{n-1}, \cos t, \sin t\right) .
$$

The reason why $\tilde{\Psi}$ is useful for our purposes is that the unit vector field $\frac{\partial}{\partial t} \in \mathfrak{X}\left(\mathbb{H}^{n} \times \mathbb{R}\right)$ is $\tilde{\Psi}$-related to the Killing vector field $\mathcal{K} \in \mathfrak{X}\left(\mathbb{R}^{n+1} \backslash \mathbb{R}^{n-1}\right)$, namely,

$$
\tilde{\Psi}_{*}(x, t) \frac{\partial}{\partial t}(x, t)=\mathcal{K}(\tilde{\Psi}(x, t))
$$

for all $x=\left(x_{0} e_{0}+\ldots+x_{n} e_{n}, t\right) \in \mathbb{H}^{n} \times \mathbb{R}$.

\subsection{The constant ratio property with respect to $\mathcal{K}$}

Submanifolds of $\mathbb{R}^{n+1} \backslash \mathbb{R}^{n-1}$ having the constant ratio property with respect to $\mathcal{K}$ can be classified as follows by using part (iii) of Lemma 3 and the conformal covering $\operatorname{map}(36)$. 
Theorem 22. Let $\phi: M^{m} \rightarrow \mathbb{H}^{n} \subset \mathbb{R}_{1}^{n+1}, m \geq 2$, be an isometric immersion of a product manifold $M^{m}=J \times N^{m-1}$ endowed with a polar metric. Write $\phi(s, x)=$ $\sum_{j=0}^{n} \phi_{j}(s, x) e_{j}$, where $e_{0}, \ldots, e_{n}$ is a pseudo-orthonormal basis of $\mathbb{R}_{1}^{n+1}$ as in (35). Then the map $f: M^{m} \rightarrow \mathbb{R}^{n+1} \backslash \mathbb{R}^{n-1}$ given by

$$
f(s, x)=\frac{1}{\phi_{0}(s, x)}\left(\phi_{1}(s, x), \ldots, \phi_{n-1}(s, x), \cos (A s), \sin (A s)\right)
$$

defines an immersion with the constant ratio property with respect to the Killing vector field $\mathcal{K}$.

Conversely, if $f: M^{m} \rightarrow \mathbb{R}^{n+1} \backslash \mathbb{R}^{n-1}, m \geq 2$, is an isometric immersion that has the constant ratio property with respect to $\mathcal{K}$, then it is either a rotational submanifold having $\mathbb{R}^{n-1}$ as axis, or $f\left(M^{m}\right)$ lies in a hyperplane that contains the subspace $\mathbb{R}^{n-1}$, or $f$ is locally given as above (globally, if the integral curves of $\mathcal{K}_{f}^{T}$ are defined on $\mathbb{R}$ ).

Proof. Since the map $f: M^{m} \rightarrow \mathbb{R}^{n+1} \backslash \mathbb{R}^{n-1}$ given by (37) is the composition $f=\tilde{\Psi} \circ \hat{f}$ of the conformal covering map (36) with an isometric immersion $\hat{f}: M^{m} \rightarrow \mathbb{H}^{n} \times \mathbb{R}$ as in Theorem 5 , the first statement follows from part (iii) of Lemma 3.

The converse also follows from the converse statement of Theorem 5 and part (iii) of Lemma 3 by noticing that rotational submanifolds having $\mathbb{R}^{n-1}$ as axis are precisely the images under the conformal covering map $\tilde{\Psi}: \mathbb{H}^{n} \times \mathbb{R} \rightarrow \mathbb{R}^{n+1} \backslash \mathbb{R}^{n-1}$ of the vertical cylinders in $\mathbb{H}^{n} \times \mathbb{R}$, whereas submanifolds of $\mathbb{R}^{n+1} \backslash \mathbb{R}^{n-1}$ that lie in a hyperplane of $\mathbb{R}^{n+1}$ containing the subspace $\mathbb{R}^{n-1}$ are the images under $\tilde{\Psi}$ of submanifolds of $\mathbb{H}^{n} \times \mathbb{R}$ that are contained in a horizontal slice of $\mathbb{H}^{n} \times \mathbb{R}$.

A more explicit description of hypersurfaces $f: M^{m} \rightarrow \mathbb{R}^{n+1} \backslash \mathbb{R}^{n-1}, m \geq 2$, with the constant ratio property with respect to $\mathcal{K}$, or equivalently, whose unit normal vector field makes a constant angle with $\mathcal{K}$, is as follows.

Corollary 23. Let $\phi: N^{n-1} \rightarrow \mathbb{H}^{n}$ be any hypersurface and let $\phi_{s}: N^{n-1} \rightarrow \mathbb{H}^{n} \subset \mathbb{R}_{1}^{n+1}$ be the family of parallel hypersurfaces to $\phi$, indexed on the open interval $J \subset \mathbb{R}$. Write $\phi_{s}(x)=\sum_{j=0}^{n} \phi_{j}(s, x) e_{j}$ where $e_{0}, \ldots, e_{n}$ is a pseudo-orthonormal basis of $\mathbb{R}_{1}^{n+1}$ as in (35). Then the map $f: M^{n}:=J \times N^{n-1} \rightarrow \mathbb{R}^{n+1} \backslash \mathbb{R}^{n-1}$, given by

$$
f(x, s)=\frac{1}{\phi_{0}(s, x)}\left(\phi_{1}(s, x), \ldots, \phi_{(n-1)}(s, x), \cos (A s), \sin (A s)\right), \quad A \neq 0,
$$

is a hypersurface with the constant ratio property with respect to $\mathcal{K}$.

Conversely, if $f: M^{n} \rightarrow \mathbb{R}^{n+1} \backslash \mathbb{R}^{n-1}, n \geq 2$, is a hypersurface that has the constant ratio property with respect to the Killing vector field $\mathcal{K}$, then either $f$ is a rotational hypersurface having $\mathbb{R}^{n-1}$ as axis, or $f\left(M^{n}\right)$ is an open subset of a hyperplane that contains $\mathbb{R}^{n-1}$, or it is locally given as above (globally, if the integral curves of $\mathcal{K}_{f}^{T}$ are defined on $\mathbb{R})$. 
Remark 24. For $n=2$, Corollary 23 says that any surface $f: M^{2} \rightarrow \mathbb{R}^{3} \backslash \mathbb{R}$ with the constant ratio property with respect to the Killing vector field $\mathcal{K}$ is either a rotational surface having $\mathbb{R}$ as axis, an open subset of a plane that contains $\mathbb{R}$, or it is locally (globally, if the integral curves of $\mathcal{K}_{f}^{T}$ are defined on $\mathbb{R}$ ) a surface $f: M^{2}:=J \times I \rightarrow \mathbb{R}^{3} \backslash \mathbb{R}$ given by

$$
f(t, s)=\frac{1}{\gamma_{0}(s, t)}\left(\gamma_{1}(s, t), \cos (A s), \sin (A s)\right), \quad A \neq 0,
$$

where $\gamma: I \rightarrow \mathbb{H}^{2} \subset \mathbb{R}_{1}^{3}, \gamma(t)=\gamma_{0}(t) e_{0}+\gamma_{1}(t) e_{1}+\gamma_{2}(t) e_{2}$, is any unit-speed curve, and

$$
\gamma(t, s)=\gamma_{0}(t, s) e_{0}+\gamma_{1}(t, s) e_{1}+\gamma_{2}(t, s) e_{2}=\cosh s \gamma(t)+\sinh s n(t)
$$

is the family of parallel curves to $\gamma$. Here $n(t) \in T_{\gamma(t)} \mathbb{H}^{2}$ is a unit vector orthogonal to $\gamma^{\prime}(t)$, and $e_{0}, e_{1}, e_{2}$ is a a pseudo-orthonormal basis $e_{0}, e_{1}, e_{2}$ of $\mathbb{R}_{1}^{3}$ such that $\left\langle e_{0}, e_{0}\right\rangle=$ $0=\left\langle e_{2}, e_{2}\right\rangle,\left\langle e_{0}, e_{2}\right\rangle=-1 / 2$ and $\left\langle e_{1}, e_{1}\right\rangle=1$.

Taking $\gamma: \mathbb{R} \rightarrow \mathbb{H}^{2} \subset \mathbb{R}_{1}^{3}$ as the horocycle

$$
\gamma(t)=e_{0}+t e_{1}+\left(t^{2}+1\right) e_{2},
$$

then $\gamma(t, s)=e^{s} e_{0}+t e^{s} e_{1}+\left(t^{2} e^{s}+e^{-s}\right) e_{2}$, and the corresponding surface $f: \mathbb{R}^{2} \rightarrow \mathbb{R}^{3} \backslash \mathbb{R}$ is given by

$$
f(t, s)=\frac{1}{e^{s}}\left(t e^{s}, \cos (A s), \sin (A s)\right)=\left(t, e^{-s} \cos (A s), e^{-s} \sin (A s)\right) \quad A \neq 0,
$$

the cylinder over the logarithmic spiral.

A remarkable example appears by starting with the curve $\gamma: \mathbb{R} \rightarrow \mathbb{H}^{2} \subset \mathbb{R}_{1}^{3}$ given for any $\sigma \in \mathbb{R}$ by

$$
\begin{aligned}
\gamma(t) & =\frac{\cosh (t / \cos \sigma)}{\cos \sigma} e_{0}+((t / \cos \sigma) \cosh (t / \cos \sigma)-\sinh (t / \cos \sigma)) e_{1} \\
& +\cos \sigma\left(\cosh (t / \cos \sigma)+(t / \cos \sigma)^{2} \cosh (t / \cos \sigma)-2(t / \cos \sigma) \sinh (t / \cos \sigma)\right) e_{2} .
\end{aligned}
$$

From $\left\langle\gamma^{\prime}(t), \gamma^{\prime}(t)\right\rangle=\frac{\sinh (t / \cos \sigma)}{\cos \sigma}$ it follows that $\frac{\left\langle\gamma^{\prime}(t), e_{2}\right\rangle}{\left\|\gamma^{\prime}(t)\right\|}=\cos \sigma$ for all $t \in \mathbb{R}$, that is, $\gamma$ is a helix in $\mathbb{R}_{1}^{3}$ with a light-like axis (see [4] for a parametrization of all helices in $\mathbb{R}_{1}^{3}$ lying in $\mathbb{H}^{2} \subset \mathbb{R}_{1}^{3}$, as well as for some of their properties). One can check that a unit vector field $n(t) \in T_{\gamma(t)} \mathbb{H}^{2}$ orthogonal to $\gamma^{\prime}(t)$ is

$$
\begin{aligned}
n(t) & =-\frac{\sinh (t / \cos \sigma)}{\cos \sigma} e_{0}+(\cosh (t / \cos \sigma)-(t / \cos \sigma) \sinh (t / \cos \sigma)) e_{1} \\
& +\cos \sigma\left(2(t / \cos \sigma) \cosh (t / \cos \sigma)-\left(1+(t / \cos \sigma)^{2}\right) \sinh (t / \cos \sigma)\right) e_{2},
\end{aligned}
$$

and that

$$
\left.\gamma(t, s)=-\frac{\cosh \rho}{\cos \sigma} e_{0}+\left(\frac{\cosh \rho}{\cos \sigma} t-\sinh \rho\right) e_{1}+\cos \sigma \cosh \rho+\frac{\cosh \rho}{\cos \sigma} t^{2}-2 t \sinh \rho\right) e_{2},
$$


where $\rho=\frac{t-s \sin \sigma}{\cos \sigma}$. The corresponding surface $f: M^{2} \rightarrow \mathbb{R}^{3} \backslash \mathbb{R}$ has the constant ratio property with respect to the Killing vector field $\mathcal{K}$ and is parametrized by

$$
f(t, s)=\left(\frac{\cos \sigma \cos s}{\cosh \rho}, \frac{\cos \sigma \sin s}{\cosh \rho}, t-\cos \sigma \tanh \rho\right),
$$

which is Dini's helicoidal surface of constant negative Gauss curvature. Therefore, Dini's surface is the image, under the conformal covering map $\Phi: \mathbb{H}^{2} \times \mathbb{R} \rightarrow \mathbb{R}^{3} \backslash \mathbb{R}$, of the surface in $\mathbb{H}^{2} \times \mathbb{R} \subset \mathbb{R}_{1}^{4}$ that is generated by starting with a helix $\gamma: \mathbb{R} \rightarrow \mathbb{H}^{2} \subset \mathbb{R}_{1}^{3} \subset \mathbb{R}_{1}^{4}$ with a light-like axis, taking a standard helix $\beta: \mathbb{R} \rightarrow \mathbb{H}^{1} \times \mathbb{R} \subset \mathbb{R}_{1}^{3}$ in a fixed normal space of $\gamma$ in $\mathbb{R}_{1}^{4}$, and then parallel translating $\beta$ along $\gamma$ with respect to the normal connection of $\gamma$.

Notice also that $t \mapsto f(t, 0)$ is a parametrization of a tractrix in a half-space of a plane containing the axis $\mathbb{R}$, which shows that a tractrix is the image of a helix in $\mathbb{H}^{2}$ with a light-like axis under the isometry between the hyperboloidal model of $\mathbb{H}^{2}$ and its half-space model.

We point out that surfaces in $\mathbb{R}^{3}$ whose unit normal vector field makes a constant angle $\theta \in[0, \pi / 2]$ with $\mathcal{K}$ have been investigated by Nistor and Munteanu in [18], who claimed that the only such surfaces were rotation surfaces with $\mathbb{R}$ as axis (corresponding to $\theta=0$ ), open subsets of planes containing $\mathbb{R}$ (corresponding to $\theta=\pi / 2$ ), the cylinder over a logarithmic spiral and Dini's helicoidal surface of constant negative curvature. In their proof of the classification of such surfaces, however, in the case $\theta \in(0, \pi / 2)$ they choose local coordinates satisfying some properties, which turn out to exist only for Dini's surface and the cylinder over a logarithmic spiral. As a consequence, their classification theorem misses the remaining surfaces given by (38) in terms of an arbitrary unit-speed curve $\gamma: I \rightarrow \mathbb{H}^{2} \subset \mathbb{R}_{1}^{3}$ other than a horocycle and a helix in $\mathbb{H}^{2}$ with a lightlike axis, which give rise to the cylinder over a logarithmic spiral and Dini's surface, respectively.

\subsection{The principal direction property with respect to $\mathcal{K}$}

The classification of submanifolds of $\mathbb{R}^{n+1} \backslash \mathbb{R}^{n-1}$ having the principal direction property with respect to $\mathcal{K}$ follows accordingly from part $(i v)$ of Lemma 3 and Theorem 9.

Theorem 25. Let $f: N^{m-1} \times I \rightarrow \mathbb{H}^{n} \times \mathbb{R} \subset \mathbb{R}_{1}^{n+2}$ be given by (22) in terms of a smooth regular curve $\gamma: I \rightarrow \mathbb{H}^{k} \times \mathbb{R} \subset \mathbb{R}_{1}^{k+2}, \gamma=\left(\gamma_{1}, \ldots, \gamma_{k+2}\right)$, such that $\gamma_{k+2}$ has nonvanishing derivative. Write $\pi \circ f: N^{m-1} \times I \rightarrow \mathbb{H}^{n} \subset \mathbb{R}_{1}^{n+1}$ as $\pi \circ f=\sum_{j=0}^{n} f_{j} e_{j}$, where $\pi: \mathbb{H}^{n} \times \mathbb{R} \rightarrow \mathbb{H}^{n}$ is the projection and $e_{0}, \ldots, e_{n}$ is a pseudo-orthonormal basis of $\mathbb{R}_{1}^{n+1}$ as in (35). Then the restriction to the subset of regular points of the map $\hat{f}: N^{m-1} \times I \rightarrow \mathbb{R}^{n+1} \backslash \mathbb{R}^{n-1}$ given by

$$
\hat{f}(x, s)=\frac{1}{f_{0}(x, s)}\left(f_{1}(x, s), \ldots, f_{n-1}(x, s), \cos \left(\gamma_{k+2}(s)\right), \sin \left(\gamma_{k+2}(s)\right)\right)
$$


is an immersion with the principal direction property with respect to $\mathcal{K}$.

Conversely, if $\hat{f}: M^{m} \rightarrow \mathbb{R}^{n+1} \backslash \mathbb{R}^{n-1}, m \geq 2$, is an isometric immersion that has the principal direction property with respect to $\mathcal{K}$, then either it is a rotational submanifold having $\mathbb{R}^{n-1}$ as axis, or its image lies in a hyperplane that contains the subspace $\mathbb{R}^{n-1}$, or it is locally given as above (globally, if the integral curves of $\mathcal{K}_{\hat{f}}^{T}$ are defined on $\mathbb{R}$ ).

If, in the above statement, the smooth regular curve $\gamma: I \rightarrow \mathbb{H}^{k} \times \mathbb{R} \subset \mathbb{R}_{1}^{k+2}$, $\gamma=\left(\gamma_{1}, \ldots, \gamma_{k+2}\right)$, is such that $\bar{\gamma}: I \rightarrow \mathbb{H}^{k} \subset \mathbb{R}_{1}^{k+1}, \bar{\gamma}=\left(\gamma_{1}, \ldots, \gamma_{k+1}\right)$, has unit speed and $\gamma_{k+2}(s)=A s$ for all $s \in I$, with $A \neq 0$, then it follows from Corollary 10 that the map $\hat{f}: M^{m} \rightarrow \mathbb{R}^{n+1} \backslash \mathbb{R}^{n-1}$ given by (39) is an immersion with both the constant ratio and principal direction properties with respect to the Killing vector field $\mathcal{K}$, and that, conversely, any isometric immersion $\hat{f}: M^{m} \rightarrow \mathbb{R}^{n+1} \backslash \mathbb{R}^{n-1}, m \geq 2$, that has both the constant ratio and principal direction properties with respect to $\mathcal{K}$ either is a rotational submanifold having $\mathbb{R}^{n-1}$ as axis, or its image lies in a hyperplane that contains the subspace $\mathbb{R}^{n-1}$, or it is locally given in this way (globally, if the integral curves of $\mathcal{K}_{\hat{f}}^{T}$ are defined on $\mathbb{R}$ ).

In the case of hypersurfaces, a more explicit description, similar to that in Corollary 23, follows as before from Theorem 25 by taking into account Lemma 6 .

\subsection{The case of conformal Killing vector fields}

Let $x_{1}, \ldots, x_{n+1}$ denote as before the standard coordinates in $\mathbb{R}^{n+1}$ and $\partial_{x_{i}}$ a unit vector field tangent to the $x_{i}$-coordinate curve, $1 \leq i \leq n+1$. The Lie algebra of conformal Killing vector fields in $\mathbb{R}^{n+1}$ has dimension $\frac{1}{2}(n+2)(n+3)$ and is generated by the Killing vector fields given by (33) and (34), by the radial vector field $\sum_{i=1}^{n+1} x_{i} \partial_{x_{i}}$, and by the vector fields

$$
\mathcal{C}_{i}=\frac{1}{2}\left(x_{i}^{2}-\sum_{j \neq i} x_{j}^{2}\right) \partial_{x_{i}}+x_{i} \sum_{j \neq i} x_{j} \partial_{x_{j}}, \quad 1 \leq i \leq n+1 .
$$

The isometric immersions into Euclidean space that have either the constant ratio or the principal direction property with respect to any of such vector fields have been described in the previous sections, except for the vector fields $\mathcal{C}_{i}$ in (40).

A description in the latter case follows from the observation that

$$
\mathcal{I}_{*} \partial_{x_{i}}=-\frac{2}{\sum_{i=1}^{n} x_{i}^{2}} \mathcal{C}_{i}, \quad 1 \leq i \leq n+1,
$$

where $\mathcal{I}$ is an inversion with respect to a unit sphere centered at the origin. Indeed, in view of (41), it follows from part (iii) (respectively, part (iv)) of Lemma 3 and Theorem 5 (respectively, Theorem 9), with $\frac{\partial}{\partial t}=\partial_{x_{i}}$, that any isometric immersion 
$f: M^{m} \rightarrow \mathbb{R}^{n} \backslash\{0\}$ with the constant ratio (respectively, principal direction) property with respect to $\mathcal{C}_{i}$ is given by $f=\mathcal{I} \circ \hat{f}$, where $\hat{f}: M^{m} \rightarrow \mathbb{R}^{n}$ is given as in Theorem 5 (respectively, Theorem 9). In the hypersurface case, a more explicit description is given by such a composition with $\hat{f}$ given as in Corollary 2 (respectively, Theorem 1) of [19]. Moreover, if $\hat{f}: M^{m} \rightarrow \mathbb{R}^{n}$ is as in Corollary 10, with $\frac{\partial}{\partial t}=\partial_{x_{i}}$, then $f=\mathcal{I} \circ \hat{f}: M^{m} \rightarrow \mathbb{R}^{n}$ has both the constant ratio and the principal direction properties with respect to $\mathcal{C}_{i}$.

\section{References}

[1] B.-Y. Chen, Constant-ratio hypersurfaces, Soochow J. Math. 27 (2001), no. 4, 353362.

[2] B.-Y. Chen, Constant-ratio space-like submanifolds in pseudo-Euclidean space, Houston J. Math. 29 (2003), no. 2, 281-294.

[3] B.-Y. Chen, Geometry of position functions of Riemannian submanifolds in pseudoEuclidean space, J. Geom. 74 (2002), no. 1-2, 61-77.

[4] M. Dajczer and R. Tojeiro, On Flat Surfaces in Space Forms, Houston J. of Math. 21 (1995), 319-338.

[5] F. Dillen, J. Fastenakels, J. Van der Veken and L. Vrancken, Constant angle surfaces in $\mathbb{S}^{2} \times \mathbb{R}$, Monatsh. Math. 152 (2007), no. 2, 89-96.

[6] F. Dillen, J. Fastenakels and J. Van der Veken, Surfaces in $\mathbb{S}^{2} \times \mathbb{R}$ with a canonical principal direction, Ann. Global Anal. Geom. 35 (2009), no. 4, 381-396.

[7] F. Dillen and M. I. Munteanu, Constant angle surfaces in $\mathbb{H}^{2} \times \mathbb{R}$, Bull. Braz. Math. Soc. (N.S.) 40 (2009), no. 1, 85-97.

[8] F. Dillen, M. I. Munteanu and A. I. Nistor, Canonical coordinates and principal directions for surfaces in $\mathbb{H}^{2} \times \mathbb{R}$, Taiwanese J. Math. 15 (2011), no. 5, 2265-2289.

[9] A. J. Di Scala and G. Ruiz-Hernández, CMC hypersurfaces with canonical principal direction in space forms, Math. Nachr. 290 (2017), no. 2-3, 248-261.

[10] E. Garnica, O. Palmas and G. Ruiz-Hernández, Hypersurfaces with a canonical principal direction, Differential Geom. Appl. 30 (2012), no. 5, 382-391.

[11] E. Garnica, O. Palmas and G. Ruiz-Hernández, Classification of constant angle hypersurfaces in warped products via eikonal functions, Bol. Soc. Mat. Mexicana (3) 18 (2012), no. 1, 29-41. 
[12] F. Dillen, M. I. Munteanu, J. Van der Veken and L. Vrancken, Classification of constant angle surfaces in a warped product, Balkan J. Geom. Appl. 16 (2) (2011), $35-47$.

[13] F. Dillen, J. Fastenakels, J. Van der Veken, Rotation hypersurfaces in $\mathbb{S}^{n} \times \mathbb{R}$ and $\mathbb{H}^{n} \times \mathbb{R}$, Note di Matematica 29 (2008), 41-54.

[14] F. Manfio and R. Tojeiro, Hypersurfaces with constant sectional curvature of $\mathbb{S}^{n} \times \mathbb{R}$ and $\mathbb{H}^{n} \times \mathbb{R}$, Illinois J. Math. 55 (1) (2011), 397-415.

[15] B. Mendonça and R. Tojeiro, Umbilical submanifolds of $\mathbb{S}^{n} \times \mathbb{R}$, Canadian J. Math. 66 (2014), 400-428.

[16] M. I. Munteanu, From golden spirals to constant slope surfaces, J. Math. Phys. 51 (7) (2010), 073507, 9 pp.

[17] M. I. Munteanu and Y. Fu, Generalized constant ratio surfaces in $\mathbb{R}^{3}$, Bull. Braz. Math. Soc. 45 (1) (2014), 73-90.

[18] M. I. Munteanu and A. I. Nistor, Surfaces in $\mathbb{E}^{3}$ making constant angle with Killing vector fields, Internat. J. Math. 23 (2012), 1250023, 16 pp.

[19] R. Tojeiro, On a class of hypersurfaces in $\mathbb{S}^{n} \times \mathbb{R}$ and $\mathbb{H}^{n} \times \mathbb{R}$, Bull. Braz. Math. Soc. 41 (2010), 199-209.

[20] R. Tojeiro, A decomposition theorem for immersions of product manifolds, Proc. Edinburgh Math. Soc. 59 (2016), 247-269.

[21] A. Yampolski, Eikonal Hypersurfaces in the Euclidean n-Space, Mediterr. J. Math. (2017) 14: 160.

Fernando Manfio and Ruy Tojeiro

Universidade de São Paulo

Instituto de Ciências Matemáticas e de Computação.

Av. Trabalhador São Carlense 400

13560-970 - São Carlos

BRAZIL

manfio@icmc.usp.br and tojeiro@icmc.usp.br

Joeri Van der Veken

KU Leuven, Department of Mathematics

Celestijnenlaan 200B - Box 2400

3001 Leuven

BELGIUM

joeri.vanderveken@kuleuven. be 